\title{
Throughput Maximization in Cloud-Radio Access Networks using Cross-Layer Network Coding
}

\author{
Mohammed S. Al-Abiad, Student Member, IEEE, Ahmed Douik, Student Member, IEEE, \\ Sameh Sorour, Senior Member, IEEE, and Md. Jahangir Hossain, Senior Member, IEEE
}

\begin{abstract}
Cloud radio access networks (C-RANs) are promising paradigms for the fifth-generation (5G) networks due to their interference management capabilities. In a C-RAN, a central processor (CP) is responsible for coordinating multiple Remote Radio Heads (RRHs) and scheduling users to their radio resource blocks (RRBs). In this paper, we develop a novel cross-layer network coding (CLNC) approach that proposes to optimize RRH's transmit powers and user's rates in making the coding decisions. As such, cross-layer throughput of the network is maximized. The joint user scheduling, file encoding, and power adaptation problem is solved by designing a subgraph for each RRB, in which each vertex represents potential user-RRH associations, encoded files, transmission rates, and power levels (PLs) for one RRB. It is then shown that the C-RAN throughput maximization problem is equivalent to a maximum-weight clique problem over the union of all such subgraphs, called herein the CRAN-CLNC graph. Numerical results revealed that the proposed joint and iterative schemes offer improved throughput performances as compared to the existing algorithms in the literature. Compared to our proposed joint scheme, our proposed iterative scheme has a certain degradation, roughly in the range of $9 \%-14 \%$. This small degradation in the throughput performance of the iterative scheme comes at the achieved low computational complexity as compared to the high complexity of the joint scheme.
\end{abstract}

Index Terms-Cloud radio access networks, coordinated scheduling, cross-layer network coding, file streaming, power allocation, timecritical applications.

\section{INTRODUCTION}

C LOUD radio access networks (C-RANs) are promising solutions to satisfy the growing traffic demand in 5G mobile networks [2]. In C-RANs, a cloud-computing central processor $(\mathrm{CP})$ is connected to multiple remote radio heads (RRHs) through fronthaul links. In the C-RAN's literature, two common strategies have been proposed, namely datasharing strategy and compression-based strategy, to efficiently use the fronthaul links [3]. In the data-sharing strategy, the $\mathrm{CP}$ splits and shares user's message with only a subset of RRHs, which encode these messages and send them over wireless channels to the users [4], [5]. The RRHs in the compression-based strategy transmit only compressed versions of the encoded messages to the users [6], [7]. Different coding schemes [8]-[11] have been studied in the literature as a means to maximize the achievable rates. For example, in [9], the authors proposed two lattice-based coding schemes that maximize the achievable sum rate in uplink multi-cell networks. In [11], the authors built on the code proposed in [10] to further improve the sum rate maximization.

- Mohammed S. Al-Abiad and Md. Jahangir Hossain are with the School of Engineering, The University of British Columbia, Kelowna, BC V1V 1V7, Canada(e-mail:m.saif@alumni.ubc.ca, jahangir.hossain@ubc.ca).

- Ahmed Douik is with the Department of Electrical Engineering, California Institute of Technology, Pasadena, CA 91125 USA (e-mail: ahmed.douik@caltech.edu).

- Sameh Sorour is with the School of Computing, Queen's University, Kingston, ON, K7L 3N6, Canada (e-mail: sameh.sorour@queensu.ca).

A part of this paper [1] is presented at the IEEE International Conference on Communications Workshops (ICCW' 2018), Kansas City, MO, USA.
Cross-layer design that uses Network Coding (NC) [12] has also been proposed to optimize different parameters in different network settings [13]-[17]. In [13], the authors used NC to maximize the sum-rate in C-RANs. The authors of [14], [15] proposed a cross-layer design for network planning in Ad-Hoc wireless networks. In [16], [17], the authors considered a cross-layer optimization and NC to optimize the throughput in wireless queuing tandem and wireless multihop networks, respectively. However, most of the existing works in C-RANs require optimization over different factors, such as RRH's beamforming vectors, user$\mathrm{RRH}$ association, and message traffic over the fronthaul links. This makes the C-RAN's design complex. In this paper, we consider the downlink of C-RANs from a different perspective. Particularly, we consider the joint target of users-RRHs scheduling policy, users' flow, and power control at the $\mathrm{CP}$, and relay them to the RRHs through lowrate fronthaul links.

In general, the existing works addressed the throughput maximization problem and its related factors individually. Particularly, works discussed in [6], [18]-[31] considered only the physical-layer factors, such as resource scheduling and power adaptation. This results in assigning a single user to each RRH's radio resource block (RRB). On the contrary, works presented in [32]-[39] considered only upper-layer factors, such as users' flow. This allocates many users to each RRB. However, in order to serve all assigned users, each RRB in each RRH adopts its transmission rate to the minimum rate of all its assigned users. As a result, the cross- 
layer throughput is degraded. Therefore, it is important to optimize cross-layer throughput by considering the abovementioned factors in C-RAN resource setting.

Motivated by the above limitations of the existing works, we consider a C-RAN resource setting wherein a set of users are required to download a set of popular files. The users are connected to a subset of RRHs and thus possess a subset of the files. We are interested in streaming these requested popular files by users to maximize cross-layer throughput. One application of this work is streaming a set of files, i.e., several videos, about an important/trending event, e.g., football game, essential updates on a famous application, etc.

\subsection{Related Works and Motivation}

The joint user scheduling and power adaptation problem is shown to be challenging [18]-[20]. Indeed, besides its joint combinatorial nature, the mathematical formulation reveals that the power adaptation itself in this setting can be non-convex. A large body of literature, see for examples, [18]-[20], [24], [25] tackled the problem by solving it iteratively. The authors of [18], [20] solved the problem via an integer relaxation method in single and multi-cell configurations, respectively, to maximize throughput. In [6], [26], the authors considered inter-cell level coordination in which only low-rate fronthaul link is used. The studies are extended in [27]-[29] to optimize the power level of each radio resource through a power optimization step. To this end, the authors of [30], [31] considered a scheduling-level coordinated C-RAN to solve the joint user scheduling and power optimization problem using graph theory techniques.

As previously stated, all studies mentioned above view the network solely from the physical-layer perspective without taking into consideration upper-layer facts. This results in assigning a single user to each RRB; e.g., a group of OFDMA sub-carrier, a TDMA timeslot, etc. However, it has been observed that users tend to have a common interest in downloading popular files, especially videos, within a small interval of time. This creates a pool of side information in the network. This side information is widely found in the current wireless networks. For example, the channel imperfections, e.g., interference, fading, etc. always cause file's erasures. The diversity in prior downloads of the popular files by different users generates side information for their new requests by others. Also, the rate-based service optimization protocols with users' achievable capacities play a significant role in generating side information. For clarification, let us consider a scenario of two groups of users where both groups are interested in popular files. The achievable capacities of the first group are much lower than the second one. The modern LTE broadcast will target the first group of users with very low rates. If we use NC, users in the first group would not be served in the current transmission's frame and left to be served on a later transmission's frame when their rates become higher. This results in generating side information. This side information can be clearly exploited using NC to maximize throughput. In NC, such side information is employed to mix users' flow and aid the coding decision at the CP. Hence, it leads to serving these users simultaneously on the same RRB.
In the recent literature, a rate-aware NC (RANC) scheme has been recommended in a number of works, see for example [37]-[39]. In the RANC scheme, not only the side information is used for NC decisions as in the classical NC, but also the rate heterogeneity of different users to different RRHs. Thus, RANC scheme has the potential to maximize the system's throughput. The authors of [38] proposed a novel joint rate selection and network coding (RSNC) scheme for increasing the system's benefit in a point-tomultipoint system (PMP). In [37] and [39], the authors proposed RANC scheme to minimize the actual completion time in PMP and C-RAN systems, respectively. However, in [39], the authors assumed that each RRH maintains at a fixed power. Moreover, the transmission rate of all RRHs is the same and will be limited by the weakest RRH, i.e., the $\mathrm{RRH}$ who can support the lowest transmission rate. This may violate the quality of service rate guarantee.

The conference version of this work [1] considered the passive RANC scheme, which requires only file combination and rate selection for scheduling decisions. The joint and iterative proposed schemes here include the set of power levels of the RRBs and their simultaneous transmission rates in decision making. Besides the fact that RANC and CRAN can be contradicting, power level employed in each RRB plays a significant role in this trade-off. In particular, increasing the power in one RRB of any RRH guarantees a high service rate for its assigned users, but also generates higher interference on the users served by the same RRB in different RRHs. Consequently, users' side information, their associations to RRH/RRB, power adaptation, rate heterogeneity, and CRAN resource setting generate highly complex interplays and trade-offs.

\subsection{Contributions}

We introduce a fully cross-layer optimization framework for capturing the above-mentioned interplays. The main contributions and results of this work can be summarized as follows.

1) We design the cross-layer optimization framework using graph-theoretic techniques, in which a novel graph, called herein the CRAN-CLNC graph. Such a CRANCLNC graph takes the following aspects into consideration.

- User Multiplexing using NC: In each vertex, subsets of users are multiplexed in each RRB of each RRH based on their side information using NC.

- Rate Adaptation: We consider an adaptive transmission rate mechanism in different vertices that represent the same RRB and the same RRH. Thus, each RRB selects the best transmission rate that is suitable for all its associated users.

- Optimal Power Allocation: We also consider a power control solution for each association that represents scheduled users to the same RRBs across all RRHs. This allows optimizing the received Signal-to-Interference-plus-Noise ratio at the different users. Hence, it improves throughput. 
2) We show that the joint coordinated scheduling and power optimization problem is equivalent to a maximum-weight clique problem over the designed CRAN-CLNC graph. Then, we solve it using a joint greedy algorithm.

3) We analyze the complexity of the joint solution. Afterward, for high complexity mitigation of the joint solution, we propose a relatively low-complexity iterative solution.

4) Selected numerical results revealed that both proposed schemes offer improved throughput performances as compared to the existing schemes. Compared to the proposed joint solution, our proposed iterative scheme has a certain degradation. This small degradation, roughly in the range of $9 \%-14 \%$, in the throughput performance of the iterative scheme comes at the achieved low computational complexity as compared to the high complexity of the joint scheme.

The rest of the paper is organized as follows. We describe the system model in Section 2. We formulate the joint problem and discuss the design of CRAN-CLNC graph in Section 3. We propose joint and iterative solutions in Section 4 and Section 5, respectively. Section 6 presents some numerical results, and Section 7 concludes the work.

\section{System Model and Parameters}

\subsection{Cloud Radio Access Network Model}

Consider the downlink communication in C-RANs in which a central processor (CP) is connected to $B$ RRHs, denoted by the set $\mathcal{B}=\left\{b_{1}, b_{2}, \cdots, b_{B}\right\}$. These RRHs are distributed in different geographic locations within a cell and cooperated in serving $U$ users, denoted by the set $\mathcal{U}=$ $\left\{u_{1}, u_{2}, \cdots, u_{U}\right\}$. The $B$ RRHs are under the CP coordination and communicate with the $\mathrm{CP}$ through low-rate fronthaul links. Similar to [30], each user and each RRH is assumed to be equipped with one single-antenna. Fig. 1 shows a C-RAN with 3 RRHs cooperating to serve 6 users simultaneously.

The transmit frame of each RRH consists of $Z$ orthogonal time/frequency RRBs that are denoted by the set $\mathcal{Z}=\left\{z_{1}, z_{2}, \cdots, z_{Z}\right\}$, as shown in Fig. 2 . Therefore, the total number of RRBs in the system is $Z_{\mathrm{tot}}=B Z$. Let $P_{b_{n} z_{m}}$ be the power allocation level (PL) of the $z_{m}$-th RRB in the $b_{n}$-th RRH. Let $\mathbf{P}=\left[P_{b_{n} z_{m}}\right]$ be a $B \times Z$ matrix containing the PLs of the RRBs/RRHs in the considered system. From practical constraints, the power level of each RRB is bounded by $P_{b_{n} z_{m}} \leqslant P_{b_{n} z_{m}}^{\max }$, where $P_{b_{n} z_{m}}^{\max }$ is the maximum power level of the RRBs in the system.

Let $h_{b_{n} z_{m}}^{u_{i}}$ be the complex channel gain from the $z_{m}$ th RRB in the $b_{n}$-th RRH to the $u_{i}$-th user. The channel is assumed to be constant during the transmission time of a single uncoded/coded file and to change from one transmission to another. In our simulation setup, we used the SUI model in which the channel information $\left\{h_{b_{n} z_{m}}^{u_{i}}\right\}_{b_{n} \in \mathcal{B}, z_{m} \in \mathcal{Z}, u_{i} \in \mathcal{U}}$ is affected by multiple factors, e.g., fading, shadowing, but the location of the user within the service area is the dominant factor. Such channel model leads to heterogeneous physical-layer rates from different RBBs/RRHs to different users. The achievable capacity of

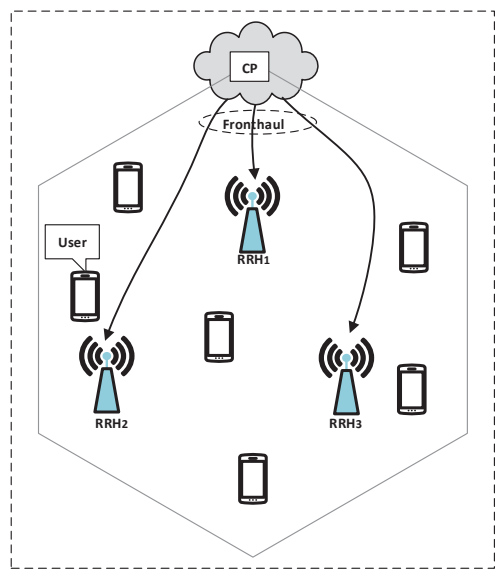

Fig. 1. A cloud radio access network composed of a central processor, 6 users, and $3 \mathrm{RRHs}$. The central processor communicates with the RRHs through low-rate fronthaul links.

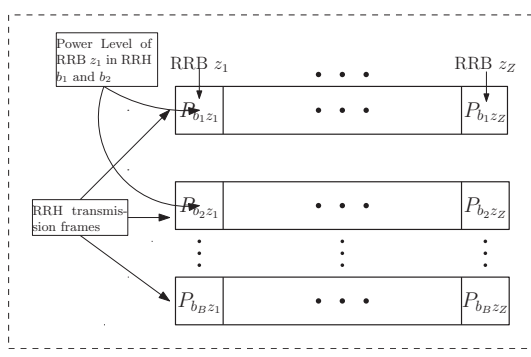

Fig. 2. Frame structure of the remote radio heads. Each RRH possesses $Z$ orthogonal radio resources synchronized with the RRBs of the remaining RRHs.

the $u_{i}$-th user assigned to the $z_{m}$-th RRB in the $b_{n}$-th RRH can be expressed as

$$
R_{b_{n} z_{m}}^{u_{i}}=\log _{2}\left(1+\operatorname{SINR}_{b_{n} z_{m}}^{u_{i}}(\mathbf{P})\right)
$$

where $\operatorname{SINR}_{b_{n} z_{m}}^{u_{i}}(\mathbf{P})$ is the corresponding signal-tointerference plus noise-ratio experienced by the $u_{i}$-th user when it is assigned with the $z_{m}$-th RRB of the $b_{n}$-th RRH, and can be expressed as

$$
\operatorname{SINR}_{b_{n} z_{m}}^{u_{i}}(\mathbf{P})=\frac{P_{b_{n} z_{m}}\left|h_{b_{n} z_{m}}^{u_{i}}\right|^{2}}{\sigma^{2}+\sum_{b_{n^{\prime}} \in \mathcal{B}, b_{n^{\prime}} \neq b_{n}}, P_{b_{n^{\prime}} z_{m}} \mid h_{\left.b_{n^{\prime} z_{m}}^{u_{i}}\right|^{2}}^{2}}
$$

where $\sigma^{2}$ is the additive white Gaussian noise (AWGN) power.

As stated earlier, the transmit frame of each RRH consists of $Z$ orthogonal RRBs. Therefore, interference at the $z_{m}$-th RRB is seen only from the same $z_{m}$-th RRB in the other RRHs. In other words, $\operatorname{SINR}_{b_{n} z_{m}}^{u_{i}}(\mathbf{P})$ depends solely on the scheduled users in $z_{m}$-th RRB across the remaining $b_{n^{\prime}} \neq b_{n}$ RRHs and the corresponding power level $P_{b_{n^{\prime}} z_{m}}$. We use the standard perfect modulation assumption. In other words, the reception of an uncoded/encoded file sent in the $z_{m^{-}}$ th RRB of the $b_{n}$-th RRH is successful at the $u_{i}$-th user if $R_{b_{n} z_{m}} \leqslant R_{b_{n} z_{m}}^{u_{i}}$, where $R_{b_{n} z_{m}}$ is the transmitted rate of that RRB. The set of achievable capacities of all users in all RRBs across all RRHs can be represented by the set

$$
\mathcal{R}=\bigotimes_{\left(b_{n}, z_{m}, u_{i}\right) \in \mathcal{B} \times \mathcal{Z} \times \mathcal{U}} R_{b_{n} z_{m}}^{u_{i}}
$$

where the symbol $\otimes$ represents the product of the set of the achievable capacities. 


\subsection{Cross-Layer Network Coding (CLNC)}

We assume that all users are interested in streaming one file or more out of a set $\mathcal{F}=\left\{f_{1}, f_{2}, \cdots, f_{F}\right\}$ containing a finite window of $F$ files. These files are deemed popular due to their previous multiple downloads by different subsets of the users over a short period of time. Popular files in this work represent frames from video-on-demand streaming. A user can start playing the video after some (short) time for buffering in $z_{m}$-th RRB, while download goes on in other $z_{m} \neq z_{m^{\prime}}$ RRBs in the same RRH. If users request more than one file simultaneously, they can get them from the same RRH by listening to multiple RRBs, each delivering one of its required files. All files in $\mathcal{F}$ are assumed to be stored in the CP with the same size of $N$ bits.

It is assumed that each RRH holds/caches the whole set of files $\mathcal{F}$ that they receive from the $\mathrm{CP}$ and updates the $\mathrm{CP}$ of the indices of the downloaded files by users after each transmission. Furthermore, the $\mathrm{CP}$, which is a high computational device, keeps a log file to temporarily store all the requested and downloaded popular files by the users under its coverage. Then, it can track the downloaded files by users and use them to do instantly decodable NC (IDNC) combinations. Finally, the log entries of users of the current popular files are transferred as part of this user's handover procedure if this user moves from one $\mathrm{CP}$ to another.

RRHs and users use the previously mentioned asymmetric users' downloaded/cached files to perform XOR encoding and decoding operations, respectively, when new files requested by users. In particular, users need the cached files to extract the wanted files immediately. The entire process of receiving and decoding these requested files takes a small duration of time. Thus, in any arbitrary scheduling epoch, the files of $\mathcal{F}$ can be classified for $u_{i}$-th user as follows.

- The Has set $\mathcal{H}_{u_{i}}$ containing files previously cached by the $u_{i}$-th user.

- The Lacks set $\mathcal{L}_{u_{i}}=\mathcal{F} \backslash \mathcal{H}_{u_{i}}$ containing the noncached files.

- The Wants set $\mathcal{W}_{u_{i}} \subset \mathcal{L}_{u_{i}}$ containing files requested by the $u_{i}$-th user in the current scheduling frame.

The CP exploits the aforementioned side information diversity to transmit encoded files in order to maximize the number of successfully received bits, i.e., throughput, in each scheduling frame. Let $\tau_{b_{n} z_{m}}$ denotes the targeted set of users benefiting from the encoded file $\kappa_{b_{n} z_{m}}$ that transmitted over the $z_{m}$-th RRB of the $b_{n}$-th RRH, where $\kappa_{b_{n} z_{m}}$ is an element of the power set $\mathcal{P}(\mathcal{F})$ representing a coded combination of a set of files. An instantly decodable combination $\kappa_{b_{n} z_{m}}$ is used to retrieve a new wanted file by $u_{i}$-th user if and only if

1) $R_{b_{n} z_{m}} \leqslant R_{b_{n} z_{m}}^{u_{i}}$ :The $u_{i}$-th user can properly receive the combination with a rate below its achievable capacity on the $z_{m}$-th RRB of the $b_{n}$-th RRH.

2) $\left|\mathcal{W}_{u_{i}} \cap \kappa_{b_{n} z_{m}}\right|=1$ : The $u_{i}$-th user can re-XOR $\kappa_{b_{n} z_{m}}$ with its previously cached files to retrieve a new file.

Therefore, the set of targeted users when the $z_{m}$-th RRB in $b_{n}$-th RRH transmits the IDNC combination $\kappa_{b_{n} z_{m}}$ is

$$
\tau_{b_{n} z_{m}}=\left\{u_{i} \in \mathcal{U}|| \mathcal{W}_{u_{i}} \cap \kappa_{b_{n} z_{m}} \mid=1 \& R_{b_{n} z_{m}} \leqslant R_{b_{n} z_{m}}^{u_{i}}\right\}
$$

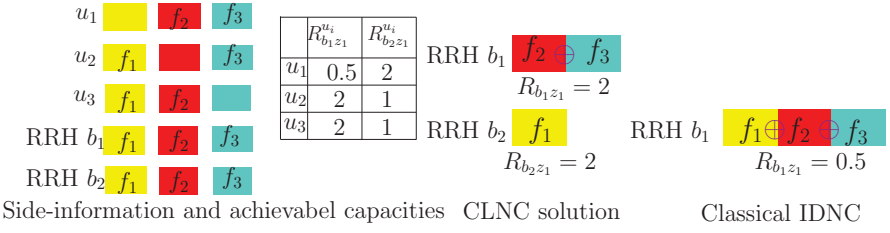

Fig. 3. A cloud radio access network composed of 3 users, 3 files, 2 remote radio heads, and $1 \mathrm{RRB}$ in each $\mathrm{R} R \mathrm{H}$ 's transmit frame.

The above-mentioned concepts can be illustrated in the following example.

Example 1: This example considers a simple model in Fig. 3 that consists of 2 RRHs, 1 RRB in each RRH's transmit frame, 3 users, users' side information and their rates. Each user $u_{i}$ possesses 2 files and wants 1 file. For example, $u_{1}$ possesses $f_{2}, f_{3}$, and wants $f_{1}$. The whole set of files is cached by each RRH. Given a certain power level to each of the RRHs, assume that users' rates in bits/s are provided in a table on the middle of Fig. 3. In order to maximize throughput for this example, many possible solutions can be found as follows.

- CLNC solution: The $b_{1}$-th RRH XORes $f_{2}$ and $f_{3}$ into $\kappa_{b_{1} z_{1}}=f_{2} \oplus f_{3}$ and transmits $\kappa_{b_{1} z_{1}}$ with a rate of 2 bits/s to the set of users $\tau_{b_{1} z_{1}}=\left\{u_{2}, u_{3}\right\}$. The $b_{2}$ th RRH transmits $\kappa_{b_{2} z_{1}}=f_{1}$ with a rate of 2 bits $/ \mathrm{s}$ to the set $\tau_{b_{2} z_{1}}=u_{1}$. Thus, $\kappa_{b_{1} z_{1}}$ in $b_{1}$-th RRH is instantly decodable for all served users $\tau_{b_{1} z_{1}}$, and $\kappa_{b_{2} z_{1}}$ in $b_{2}$-th RRH is instantly decodable only for $u_{1}$ in $\tau_{b_{2} z_{1}}$. Given this, we have the follwoing decoding process at the users. User $u_{2}$ gets file $f_{2}$ by XORing $\kappa_{b_{1} z_{1}}$ with file $f_{3}$ and user $u_{3}$ gets file $f_{3}$ by XORing $\kappa_{b_{1} z_{1}}$ with file $f_{2}$. Therefore, the best achievable overall throughput in this scenario is 6 bits/s as each served user will simultaneously receive 2 bits/s.

- Uncoded solution: The $b_{1}$-th and $b_{2}$-th RRHs transmit $f_{2}$ and $f_{1}$ with an equal rate of 2 bits/s, respectively, to $u_{2}$ and $u_{1}$. This results in 4 bits/s throughput achievable.

- Classical IDNC solution: The $b_{1}$-th RRH XORes $f_{1}$, $f_{2}$, and $f_{3}$ into $\kappa_{b_{1} z_{1}}=f_{1} \oplus f_{2} \oplus f_{3}$ and transmits $\kappa_{b_{1} z_{1}}$ to the set of users $\tau_{b_{1} z_{1}}=\left\{u_{1}, u_{2}, u_{3}\right\}$. This can be done from the network coding viewpoint but will degrade the throughput than the uncoded and CLNC solutions. Indeed, to serve all users given their files are combined using $\mathrm{NC}$, this solution will limit the transmitting rate to $0.5 \mathrm{bit} / \mathrm{s}$, resulting in total throughput of 1.5 bits $/ \mathrm{s}$.

From the completion time viewpoint, CLNC needs a total of $N$ seconds, the uncoded solution consumes $1.5 \mathrm{~N}$ seconds, and classical IDNC requires $2 N$ seconds. This clearly shows that CLNC is more efficient in maximizing the throughput and minimizing the completion time. These results can be further improved by optimizing the power allocation for each RRB in each RRH.

\section{Problem formulation and Graph Con- STRUCTION}

\subsection{Problem Formulation}

The joint user scheduling and power optimization problem considered in this paper is equivalent to assign users to the 
RRBs of the RRHs and adapt the power levels under the following network connectivity constraints (CC).

- CC1: Each user can connect to at most one RRH, but possibly to many RRBs in that RRH.

- CC2: Each power level PL is bounded by a value.

Let $X_{b_{n} z_{m}}^{u_{i}}$ be a binary variable that is equal to 1 if $u_{i}$ th user is assigned to $z_{m}$-th RRB of $b_{n}$-th RRH, and zero otherwise. Typically, $X_{b_{n} z_{m}}^{u_{i}}$ will be 0 if $b_{n}$-th RRH does not store the requested file by $u_{i}$-th user. Let $Y_{b_{n}}^{u_{i}}$ be a binary variable that is set to 1 if $u_{i}$-th user is assigned to $b_{n}$-th $\mathrm{RRH}$, and zero otherwise. The joint coordinated scheduling and power allocation problem can be formulated as follows

$\max \sum_{b_{n} \in \mathcal{B}} \sum_{z_{m} \in \mathcal{Z}} \sum_{u_{i} \in \mathcal{U}} X_{b_{n} z_{m}}^{u_{i}} \min _{u_{i^{\prime}} \in \tau_{b_{n^{\prime}}} z_{m^{\prime}}} \log _{2}\left(1+\operatorname{SINR}_{b_{n^{\prime}} z_{m^{\prime}}}^{u_{i^{\prime}}}(\mathbf{P})\right)$

s.t. $Y_{b_{n}}^{u_{i}}=\min \left(\sum_{z_{m}} X_{b_{n} z_{m}}^{u_{i}}, 1\right),\left(b_{n}, u_{i}\right) \in \mathcal{B} \times \mathcal{U}$,

$\sum_{b_{n}} Y_{b_{n}}^{u_{i}} \leqslant 1, u_{i} \in \mathcal{U}$,

$0 \leq P_{b_{n} z_{m}} \leq P_{b_{n} z_{m}}^{\max },\left(b_{n}, z_{m}\right) \in \mathcal{B} \times \mathcal{Z}$,

$X_{b_{n} z_{m}}^{u_{i}}, Y_{b_{n}}^{u_{i}} \in\{0,1\}, \kappa_{b_{n} z_{m}} \in \mathcal{P}(\mathcal{F}),\left(u_{i}, b_{n}, z_{m}\right) \in \mathcal{U} \times \mathcal{B} \times \mathcal{Z}$,

where the optimization is carried over the variables $X_{b_{n} z_{m}}^{u_{i}}$ $Y_{b_{n}}^{u_{i}}, \kappa_{b_{n} z_{m}}, R_{b_{n} z_{m}}$, and $P_{b_{n} z_{m}}$. The variables $X_{b_{n} z_{m}}^{u_{i}}$ and $Y_{b_{n}}^{u_{i}}$ are discrete optimization parameters that represent the user-RRH and user-RRB associations, respectively. On the other hand, the variables $\kappa_{b_{n} z_{m}}, R_{b z}$ and $P_{b_{n} z_{m}}$ account for the file combination, the transmission rate, and the PLs for the $z_{m}$-th RRB of the $b_{n}$-th RRH, respectively. Constraints (5b) and (5c) translate CC1. Constraint (5d) corresponds to constraint CC2.

The problem in (5) is NP-hard. To show its hardness, we consider a simpler case when all users have the same rates to all RRBs/RRHs. This makes the objective function constant. In this case, the problem in (5) is reduced to be an NC problem, which is shown to be NP [40]. Therefore, the problem in (5) has the worst case exponential solution but can be solved using the branch and bound algorithm whose performance depends on the quality of lower and upper bounds. To reach a tractable solution to (5), one can discretize the continuous power $P_{b_{n} z_{m}}$ into a set of discrete PLs $\mathbf{P}$ while constructing the CRAN-CLNC graph simultaneously.

\subsection{Power Control Subgraph Configuration}

In this subsection, we first generate all possible IDNC file combinations. Then, we configure the power control subgraph for each $z_{m}$-th RRB in the network.

Let $\mathcal{S}$ be the set of all possible associations between users and files (pairs of users and their requested files), which is defined by $\mathcal{S}=\mathcal{U} \times \mathcal{F}$, and $s$ is an element in $\mathcal{S}$. For example, the set of all possible associations between users and their requested files in Example 1 is $\mathcal{S}=\left\{\left(u_{1}, f_{1}\right),\left(u_{2}, f_{2}\right),\left(u_{3}, f_{3}\right)\right\}, s=\left(u_{1}, f_{1}\right)$ represents the association of $u_{1}$-th user and its $f_{1}$-th requested file. For simplicity, $u_{i}(s)$ represents the $u_{i}$-th user in the $s$-th association and $|\mathcal{S}|$ represents the number of elements of the set $\mathcal{S}$. The generation of the set of the possible IDNC file
TABLE 1

Variables and parameters of the system

\begin{tabular}{|l|l|}
\hline Variable & Definition \\
\hline $\mathcal{U}$ & Set of $U$ users \\
\hline $\mathcal{F}$ & Set of $F$ files \\
\hline $\mathcal{B}$ & Set of $B$ Remote Radio Heads (RRHs) \\
\hline $\mathcal{Z}$ & Set of $Z$ Radio Resource Blocks (RRBs) \\
\hline $\mathcal{R}$ & Set of all achievable capacities \\
\hline $\mathcal{W}_{u_{i}}$ & Set of wanted files by $u_{i}$-th user \\
\hline$R_{b_{n}} z_{m}$ & Transmission rate of $z_{m}$-th RRB in $b_{n}$-th RRH \\
\hline$\kappa_{b_{n}} z_{m}$ & The encoded file of $z_{m}$-th RRB in $b_{n}$-th RRH \\
\hline$\tau_{b_{n} z_{m}}$ & $\begin{array}{l}\text { Set of targeted users by } z_{m} \text {-th RRB in } b_{n} \text {-th } \\
\text { RRH }\end{array}$ \\
\hline $\mathcal{S}_{\text {IDNC }}$ & Set of all possible IDNC file combinations \\
\hline $\mathcal{A}$ & Set of all possible associations in the network \\
\hline $\mathcal{A}_{z_{m}}$ & Set of all possible associations for $z_{m}$-th RRB \\
\hline
\end{tabular}

combinations, denoted by $\mathcal{S}_{\mathrm{IDNC}}$, relies on the fact that the corresponding users in the combined associations in $\mathcal{S}$ can be instantly served by one IDNC file. Therefore, any two distinct associations $s \in \mathcal{S}$ and $s^{\prime} \in \mathcal{S}$ are combined if one of the following conditions is satisfied.

- C1: $u_{i}(s) \neq u_{i}\left(s^{\prime}\right)$ and $f_{k}(s)=f_{k}\left(s^{\prime}\right)$. The two associations $s, s^{\prime}$ are induced by the same file $f_{k}$ that is requested by two distinct users.

- C2: $u_{i}(s) \neq u_{i}\left(s^{\prime}\right)$ and $f_{k}(s) \in \mathcal{H}_{u_{i}\left(s^{\prime}\right)}$ and $f_{k}\left(s^{\prime}\right) \in$ $\mathcal{H}_{u_{i}(s)}$. This represents that two different files are wanted by two different users. Meanwhile, each wanted file in each association is in the Has set of the user that induced the other association.

These conditions ensure that any IDNC file combination in $\mathcal{S}_{\text {IDNC }}$ is always decodable for the users represented by the corresponding combined associations. Therefore, $\mathcal{S}_{\text {IDNC }}$ consists of all possible IDNC file combinations resulted from the associations in $\mathcal{S}$, and $\mathbf{s}=(\kappa, \tau)$ is an element in $\mathcal{S}_{\text {IDNC, }}$ where $\kappa$ and $\tau$ are defined in Section 2.2. For example, associations $s=\left(u_{1}, f_{1}\right) \in \mathcal{S}$ and $s^{\prime}=\left(u_{2}, f_{2}\right) \in \mathcal{S}$ satisfy $\mathrm{C} 1$ and $\mathbf{C} 2$, and accordingly, we can combine them into $\mathbf{s}=(\kappa, \tau)=\left\{\left(f_{1} \oplus f_{2}\right),\left(u_{1}, u_{2}\right)\right\}$.

Let $\mathcal{A}$ be the set of all possible associations between RRHs, RRBs, IDNC file combinations, and achievable capacities, i.e., $\mathcal{A}=\mathcal{B} \times \mathcal{Z} \times \mathcal{S}_{\text {IDNC }} \times \mathcal{R}$. For example, one possible association in $\mathcal{A}$ is $\left(b_{n}, z_{m}, \mathbf{s}, R\right)$ which represents the RRH $b_{n}, \operatorname{RRB} z_{m}$, IDNC combination $\mathrm{s}$, and rate $R$. Basically, each element in $\mathcal{A}$ contains combined associations of $s \in \mathcal{S}$ representing an IDNC combination $\mathbf{s}$ that is transmitted from RRH $b_{n}$ on RRB $z_{m}$ with its corresponding rate $R$. Let $\mathcal{A}_{z_{m}} \subset \mathcal{A}$ represent the associations relative to the $z_{m}$ th RRB across all RRHs. In other words, $\mathcal{A}_{z_{m}}$ represents all associations in $\mathcal{A}$ that are indexed by $z_{m}$. The used notations and variables are summarized in Table 1.

Now let the power control subgraph of the $z_{m}$-th RRB in the network be denoted by $\mathcal{G}_{z_{m}}\left(\mathcal{V}_{z_{m}}, \mathcal{E}_{z_{m}}\right)$ wherein $\mathcal{V}_{z_{m}}$ and $\mathcal{E}_{z_{m}}$ refer to the set of vertices and edges of this subgraph, respectively. The set of vertices in the $\mathcal{G}_{z_{m}}$-th subgraph is generated by merging all possible associations in $\mathcal{A}_{z_{m}}$ for the different RRHs under the system condition CC1. Any possible merged associations in $\mathcal{A}_{z_{m}}$ is referred to as feasible schedule and denoted by $\mathbf{S}$. In $\mathbf{S}$, the same associated user is not scheduled to different RRHs as stated in the 
condition CC1. The set of all possible feasible schedules $\mathbf{S}$ corresponding to the $z_{m}$-th RRB is represented by $\mathcal{S}_{z_{m}, \mathrm{fs}}$. Therefore, a vertex $v \in \mathcal{V}_{z_{m}}$ associated with $\mathbf{S} \in \mathcal{S}_{z_{m}, \mathrm{fs}}$ satisfies $b_{n}(s) \neq b_{n}\left(s^{\prime}\right)$ and $u_{i}(s) \neq u_{i}\left(s^{\prime}\right)$ for all $s \neq s^{\prime} \in \mathbf{S}$. As a result, each vertex $v \in \mathcal{V}_{z_{m}}$ represents the partial schedule of users to the $z_{m}$-th RRB across all RRHs.

As mentioned above, the set of all vertices $\mathcal{E}_{z_{m}}$ represents the set of all merged associations. Therefore, each $v \in \mathcal{V}_{z_{m}}$ representing the feasible schedule $\mathbf{S} \in \mathcal{S}_{z_{m} \text {, fs }}$ satisfies the following local conditions (LC).

- LC1: For all $\left(s, s^{\prime}\right) \in \mathbf{S}$ such that $b_{n}(s)=b_{n}\left(s^{\prime}\right)$, we have $R(s)=R\left(s^{\prime}\right)$. This condition guarantees that all associations in the same RRB $z_{m}$ and RRH $b_{n}$ have the same transmission rate.

- LC2: For all $\left(s, s^{\prime}\right) \in \mathbf{S}$ such that $b_{n}(s) \neq b_{n}\left(s^{\prime}\right)$, we have $\tau \cap \tau^{\prime}=\varnothing$. This condition guarantees that each user is scheduled to at most a single RRH.

Suppose that the power distribution $\mathbf{P}$ will be computed later, then a vertex $v$ representing the schedule $\mathbf{S}$ has a weight that reflects the total contribution of the vertex to the network. This weight can be expressed as

$$
w(v)=\sum_{s \in \mathbf{S}} \min _{u_{i}(s) \in \tau_{b_{n}(s) z_{m}(s)}} \log _{2}\left(1+\operatorname{SINR}_{b_{n}(s) z_{m}(s)}^{u_{i}(s)}(\mathbf{P})\right) .
$$

\subsection{CRAN-CLNC Graph Design}

We design the CRAN-CLNC graph $\mathcal{G}(\mathcal{V}, \mathcal{E})$ by generating the $Z$ power control subgraphs. Without loss of generality, the set of all schedules in the network is the union of schedules of all RRBs, i.e., $\mathcal{S}_{\mathrm{fs}}=\bigcup_{z_{m} \in \mathcal{Z}} \mathcal{S}_{z_{m}}$,fs. Therefore, the corresponding vertex set of the CRAN-CLNC graph is simply the union of vertices of all the power control subgraphs, i.e., $\mathcal{V}=\bigcup_{z_{m} \in \mathcal{Z}} \mathcal{V}_{z_{m}}$. Since we already described the edges between vertices within each subgraph $\mathcal{G}_{z_{m}}$ in the previous subsection, the rest of this section describes remaining edges corresponding to different subgraphs.

Following similar philosophy as before, two different vertices belonging to two different subgraphs are adjacent if their combination results in a feasible schedule. In particular, two vertices are connected if no user is scheduled to different RRHs. To mathematically formulate this, let vertex $v \in \mathcal{G}_{z_{m}}$ be corresponding to the schedule $\mathbf{S} \in \mathcal{S}_{z_{m}, \mathrm{fs}}$ and vertex $v^{\prime} \in \mathcal{G}_{z_{m^{\prime}}}$ corresponding to the schedule $\mathbf{S}^{\prime} \in \mathcal{S}_{z_{m^{\prime}}, \text { fs }}$. Vertices $v$ and $v^{\prime}$ are adjacent if the associations they represent satisfy the following general condition (GC).

- GC: For all $\left(s, s^{\prime}\right) \in \mathbf{S} \times \mathbf{S}^{\prime}$ such that $\tau \cap \tau^{\prime} \neq \varnothing$, we have $b_{n}(s)=b_{n}\left(s^{\prime}\right)$. The condition guarantees that the same user can be associated only to a unique RRH.

The design of the CRAN-CLNC graph $\mathcal{G}$ makes any maximal clique satisfies the following criterion.

- All targeted users can retrieve a new file(s) from the transmission schedule of all RRBs and RRHs.

- Each user is scheduled to a single RRH.

- Each RRB identified by the vertices in a maximal clique adopts the transmission rate identified by the vertex. This rate supports the smallest channel capacity of all scheduled users to that RRB.
Given the optimal power allocation $\mathbf{P}$, the following theorem reformulates the problem (5).

Theorem 1. The CRAN joint coordinated scheduling and power allocation problem (5) is equivalent to a maximum-weight clique problem over the CRAN-CLNC graph, and can be written as

$$
\arg \max _{\mathbf{C} \in \mathbf{C}} \sum_{v \in \mathbf{C}} w(v),
$$

where $\mathbf{C}$ is a maximal clique in the CRAN-CLNC graph, $\mathrm{C}$ is the set of all possible maximal cliques of degree $Z$, and $w(v)$ is defined in (6). The vertices in $\mathbf{C}$ represents the targeted users and the file combinations in each RRB across all RRHs.

For proof, please refer to Appendix A.

\section{Joint Scheduling and Power Allocation SOLUTION USING CLNC}

In this section, we present the joint CRAN-CLNC solution to (5). The idea is to solve the power control problem for each vertex of the power control subgraph. This would allow the design of the CRAN-CLNC graph. Afterward, we heuristically solve the reformulated problem in Theorem 1 using greedy graph algorithm.

\subsection{Optimal Scheduling and Power Control Solution}

We discretize a set of power levels to solve (5) efficiently. More specifically, we show that by simultaneously computing the optimal power allocations $\mathbf{P}$ while designing the CRAN-CLNC graph, we can achieve a tractable solution to (5).

Consider the $z_{m}$-th power control subgraph and a vertex $v \in \mathcal{V}_{z_{m}}$ in that subgraph is associated with the schedule $\mathbf{S}=\left\{s_{1}, s_{2}, \cdots, s_{|\mathbf{S}|}\right\} \in \mathcal{S}_{z_{m}, \text { fs }}$, where $|\mathbf{S}|$ is the degree of $\mathbf{S}$, i.e., $|\mathbf{S}|=\sum_{b_{n} \in \mathcal{B}}\left|\tau_{b_{n} z_{m}}\right|$. The optimal power levels PLs $\left(P_{b_{1} z_{m}}, \cdots, P_{b_{B} z_{m}}\right)$ that maximize the throughput for that vertex $v$ are the solution to the following problem

$$
\begin{aligned}
& \max _{P_{b_{n} z_{m}}} \sum_{b_{n} \in \mathcal{B}}\left|\tau_{b_{n} z_{m}}\right| * \min _{u_{i} \in \tau_{b_{n} z_{m}}} \log _{2}\left(1+\operatorname{SINR}_{b_{n} z_{m}}^{u_{i}}(\mathbf{P})\right) \\
& \text { s.t. } 0 \leqslant P_{b_{n} z_{m}} \leqslant P_{b_{n} z_{m}}^{\max }, \forall b_{n} \in \mathcal{B},
\end{aligned}
$$

where the optimization is over the power levels $P_{b_{n} z_{m}}$, $\forall b_{n} \in \mathcal{B}$.

The power optimization problem (8) is a well-known non-convex problem [43] and can be solved optimally [44]. However, such a solution requires a high computation complexity and converges slowly. To find a reasonable solution to (8) with a low computational load, we use one of the power optimization algorithms proposed in [43].

\subsection{Greedy Algorithm for Scheduling and Power Adap- tation}

The maximum-weight clique problem in Theorem 1 is NPhard. However, we use one of the existing algorithms in the literature, e.g., [45]-[47] to solve problem (5) heuristically for one particular transmission. The proposed algorithm selects a number of vertices with the maximum weights, wherein each vertex's weight represents the sum of transmission rates of the $z_{m}$-th RRB across all RRHs that suitable for only a subset of associated users.

The joint coordinated scheduling and power allocation algorithm can be implemented at the $\mathrm{CP}$ as follows. 


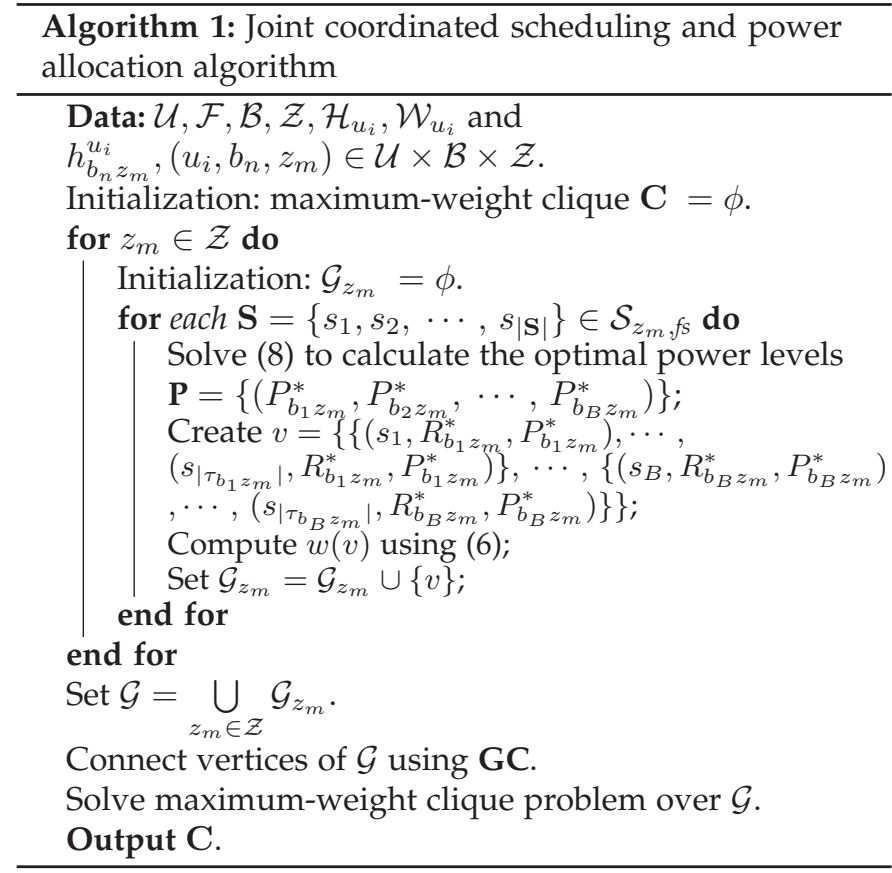

First, we design the CRAN-CLNC graph as follows. For each RRB $z_{m} \in \mathcal{Z}$, we configure the power control subgraph $\mathcal{G}_{z_{m}}$ by generating each vertex $v \in \mathcal{G}_{z_{m}}$ corresponding to the feasible schedule $\mathbf{S} \in \mathcal{S}_{z_{m} \text {,fs }}$ using conditions LC1 and LC2. Afterward, we calculate the optimal PLs for each vertex by solving the optimization problem (8). Then, we append the computed PLs of each vertex and the corresponding rates to that vertex, as explained in Section 4.1. We repeat steps above for all RRBs $z_{m} \in \mathcal{Z}$. We merge all subgraphs of all RRBs and connect them using the connection edge condition GC.

Second, in each iteration, we find the maximum-weight clique among all other maximal cliques in CRAN-CLNC graph $\mathcal{G}$, which can be implemented as follows. We compute the vertices' weights using (6). Then, we select the maximum-weight vertex $v^{*}$ and add it to $\mathbf{C}$, i.e., $\mathbf{C}$ is initially empty. Then, we update the graph $\mathcal{G}$ by removing $v^{*}$ and all the vertices that are not adjacent to it so as the next chosen vertex is not in feasible transmission conflict with the already chosen ones in $\mathbf{C}$. We execute this process until no more vertices exist in $\mathcal{G}$. Clearly, the number of vertices in the selected maximum-weight clique is $Z$. The detailed procedures of the algorithm are provided in Algorithm 1.

Given the fact that deriving the performance expressions for the considered problem in this paper is highly complex, we only used heuristic schemes. The inability to derive such expressions is widely found in existing works in the literature on a simpler yet related NC problems, e.g., [34], [37], [39], [41], [42].

\subsection{Illustration of the Proposed Algorithm}

We illustrate here how to use Algorithm 1 to design the CRAN-CLNC graph shown in Fig. 4 of the C-RAN model presented in Example 1. In Example 1, a simple C-RAN composed of 2 RRHs, 1 RRB, 3 users, and 3 files. A stepby-step illustration is given as follows.

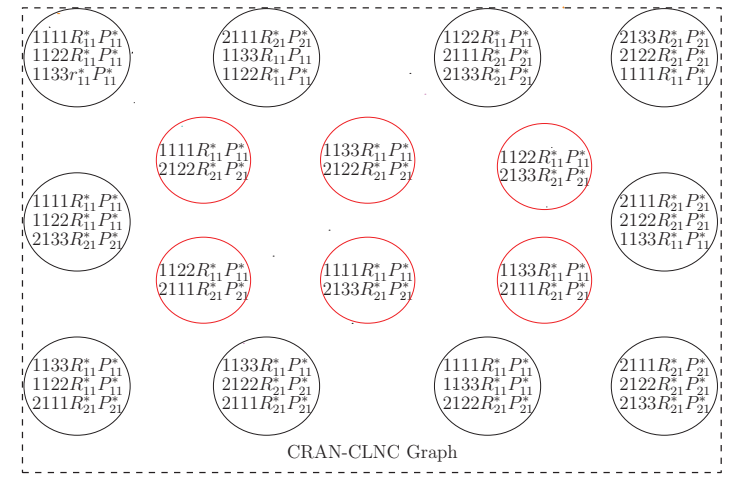

Fig. 4. CRAN-CLNC graph of Example 1 that presented in Fig. 3 based on Algorithm 1.

First step: We generate the set of all possible associations between users and their requested files, i.e., $\mathcal{S}=$ $\left\{\left(u_{1}, f_{1}\right),\left(u_{2}, f_{2}\right),\left(u_{3}, f_{3}\right)\right\}$. Then, based on the instant decodability conditions $\mathbf{C} \mathbf{1}$ and $\mathbf{C} 2$ that explained in Section 3.2 , we generate all IDNC file combinations $\mathcal{S}_{\text {IDNC }}$ as summarized in Table 2.

Second step: We generate all feasible schedules $\mathbf{S}_{i} \in \mathcal{S}_{z_{1}, \mathrm{fs}}$, $\forall i=1,2, \cdots,\left|\mathcal{S}_{z_{1}, \mathrm{fs}}\right|$, such that each schedule $\mathbf{S}$ consists of many associations $s$, in which they satisfy $\mathbf{C C} 1$. Thus, $\mathbf{S}_{i}$ is represented by a vertex $v_{i}$ in the power control subgraph as described in Section 3.2. Table 3 summarizes all these feasible schedules $\mathcal{S}_{z_{1} \text {,fs }}$.

Third step: We solve the power problem for each vertex $v_{i}$ as explained in Section 4.1. Then, we use the optimal power distribution $\mathbf{P}$ to calculate the weight of that vertex $v_{i}$ as in (6). This weight reflects the total contribution of the vertex to the network. These vertices and their corresponding weights are illustrated in Table 4 . A vertex $v_{i}$ in Table 4 contains a set of associations each one labeled by the subscripts of RRH $b_{n}$, RRB $z_{m}$, user $u_{i}$, file $f_{k}$, achievable capacity $R_{b_{n} z_{m}}^{*}$, and PL $P_{b_{n} z_{m}}^{*}$. For example, the association $1111 R_{11}^{*} P_{11}^{*}$ represents the subscripts of $b_{1}$-th RRH, $z_{1}$-th RRB, $u_{1}$-th user and its $f_{1}$-th requested file, adopted transmission rate $R_{b_{1} z_{1}}^{*}$, and power level $P_{b_{1} z_{1}}^{*}$. The first vertex has two associated users to RRB $z_{1}$ in RRH $b_{1}$ and one associated user to RRB $z_{1}$ in RRH $b_{2}$. Thus, the corresponding weight is $2 * R_{11}^{*}+R_{21}^{*}$ bits /s. The CRAN-CLNC graph that contains all configured vertices is shown in Fig. 4 . Since the C-RAN model that presented in Example 1 contains only one RRB, the constructed CRAN-CLNC graph in Fig. 4 contains only one power control subgraph and does not contain any edge connection.

Fourth step: We execute the maximum-weight clique algorithm in the CRAN-CLNC graph to find the best clique that provides an efficient solution. It is important to note that the proposed solution does guarantee the uncoded [30] solution. For example, if the best schedule that maximizes the received throughput assigns a single user to each $\mathrm{RRB} / \mathrm{RRH}$, then the proposed algorithm would provide such schedules, i.e., red color circles in Fig. 4.

\subsection{Complexity of the Joint Solution}

In this subsection, we analyze the complexity of the joint solution, which is divided into two phases: i) the complexity of constructing the CRAN-CLNC graph, and ii) the complexity 
TABLE 2

All possible IDNC combinations $\mathcal{S}_{\text {IDNC }}$

\begin{tabular}{|c|c|}
\hline $\mathrm{i}$ & $\mathbf{s}_{i}=\left(\kappa_{i}, \tau_{i}\right)$ \\
\hline \hline 1 & $\left(\left(f_{1} \oplus f_{2}\right),\left(u_{1}, u_{2}\right)\right)$ \\
\hline 2 & $\left(\left(f_{1}\right),\left(u_{1}\right)\right)$ \\
\hline 3 & $\left(\left(f_{1} \oplus f_{3}\right),\left(u_{1}, u_{3}\right)\right)$ \\
\hline 4 & $\left(\left(f_{2}\right),\left(u_{2}\right)\right)$ \\
\hline 5 & $\left(\left(f_{2} \oplus f_{3}\right),\left(u_{2}, u_{3}\right)\right)$ \\
\hline 6 & $\left(\left(f_{3}\right),\left(u_{3}\right)\right)$ \\
\hline 7 & $\left(\left(f_{1} \oplus f_{2} \oplus f_{3}\right),\left(u_{1}, u_{2}, u_{3}\right)\right)$ \\
\hline
\end{tabular}

TABLE 3

All feasible schedules $\mathcal{S}_{z_{1} \text {,fs }}$

\begin{tabular}{|c|c||c|c|}
\hline $\mathrm{i}$ & $\mathbf{S}_{i}$ & $\mathrm{i}$ & $\mathbf{S}_{i}$ \\
\hline \hline 1 & $\left\{b_{1} z_{1} \mathbf{s}_{1} R_{b_{1} z_{1}}, b_{2} z_{1} \mathbf{s}_{6} R_{b_{2} z_{1}}\right\}$ & 9 & $\left\{b_{2} z_{1} \mathbf{s}_{1} R_{b_{2} z_{1}}, b_{1} z_{1} \mathbf{s}_{6} R_{b_{1} z_{1}}\right\}$ \\
\hline 2 & $\left\{b_{1} z_{1} \mathbf{s}_{5} R_{b_{1}} z_{1}, b_{2} z_{1} \mathbf{s}_{2} R_{b_{2}} z_{1}\right\}$ & 10 & $\left\{b_{2} z_{1} \mathbf{s}_{7} R_{b_{2} z_{1}}\right\}$ \\
\hline 3 & $\left\{b_{1} z_{1} \mathbf{s}_{7} R_{b_{1} z_{1}}\right\}$ & 11 & $\left\{b_{1} z_{1} \mathbf{s}_{2} R_{b_{1} z_{1}}, b_{2} z_{1} \mathbf{s}_{4} R_{b_{2} z_{1}}\right\}$ \\
\hline 4 & $\left\{b_{2} z_{1} \mathbf{s}_{2} R_{b_{2} z_{1}}, b_{1} z_{1} \mathbf{s}_{6} R_{b_{1} z_{1}}\right\}$ & 12 & $\left\{b_{1} z_{1} \mathbf{s}_{6} R_{b_{1} z_{1}}, b_{2} z_{1} \mathbf{s}_{4} R_{b_{2} z_{1}}\right\}$ \\
\hline 5 & $\left\{b_{1} z_{1} \mathbf{s}_{6} R_{b_{1} z_{1}}, b_{2} z_{1} \mathbf{s}_{1} R_{b_{2} z_{1}}\right\}$ & 13 & $\left\{b_{1} z_{1} \mathbf{s}_{4} R_{b_{1} z_{1}}, b_{2} z_{1} \mathbf{s}_{2} R_{b_{2} z_{1}}\right\}$ \\
\hline 6 & $\left\{b_{1} z_{1} \mathbf{s}_{4} R_{b_{1} z_{1}}, b_{2} z_{1} \mathbf{s}_{3} R_{b_{2} z_{1}}\right\}$ & 14 & $\left\{b_{2} z_{1} \mathbf{s}_{6} R_{b_{2} z_{1}}, b_{1} z_{1} \mathbf{s}_{2} R_{b_{1} z_{1}}\right\}$ \\
\hline 7 & $\left\{b_{1} z_{1} \mathbf{s}_{3} R_{b_{1} z_{1}}, b_{2} z_{1} \mathbf{s}_{4} R_{b_{2} z_{1}}\right\}$ & 15 & $\left\{b_{2} z_{1} \mathbf{s}_{6} R_{b_{2} z_{1}}, b_{1} z_{1} \mathbf{s}_{4} R_{b_{1} z_{1}}\right\}$ \\
\hline 8 & $\left\{b_{2} z_{1} \mathbf{s}_{5} R_{b_{2} z_{1}}, b_{1} z_{1} \mathbf{s}_{6} R_{b_{1} z_{1}}\right\}$ & 16 & $\left\{b_{1} z_{1} \mathbf{s}_{6} R_{b_{1} z_{1}}, b_{2} z_{1} \mathbf{s}_{2} R_{b_{2} z_{1}}\right\}$ \\
\hline
\end{tabular}

TABLE 4

The generated vertices and their corresponding weights

\begin{tabular}{|l|l|l|}
\hline $\mathrm{i}$ & $v_{i}$ & $w_{i}$ \\
\hline \hline 1 & $\left\{1111 R_{11}^{*} P_{11}^{*}, 1122 R_{11}^{*} P_{11}^{*}, 2133 R_{21}^{*} P_{21}^{*}\right\}$ & $2 * R_{11}^{*}+R_{21}^{*}$ \\
\hline 2 & $\left\{1122 R_{11}^{*} P_{11}^{*}, 1133 R_{11}^{*} P_{11}^{*}, 2111 R_{21}^{*} P_{21}^{*}\right\}$ & $2 * R_{11}^{*}+R_{21}^{*}$ \\
\hline 3 & $\left\{1111 R_{11}^{*} P_{11}^{*}, 1122 R_{11}^{*} P_{11}^{*}, 1133 R_{11}^{*} P_{11}^{*}\right\}$ & $3 * R_{11}^{*}$ \\
\hline 4 & $\left\{2111 R_{21}^{*} P_{21}^{*}, 1122 R_{11}^{*} P_{11}^{*}, 1133 R_{11}^{*} P_{11}^{*}\right\}$ & $R_{21}^{*}+2 * R_{11}^{*}$ \\
\hline 5 & $\left\{1133 R_{11}^{*} P_{11}^{*}, 2122 R_{21}^{*} P_{21}^{*}, 2111 R_{21}^{*} P_{21}^{*}\right\}$ & $R_{11}^{*}+2 * R_{21}^{*}$ \\
\hline 6 & $\left\{1122 R_{11}^{*} P_{11}^{*}, 2111 R_{21}^{*} P_{21}^{*}, 2133 R_{21}^{*} P_{21}^{*}\right\}$ & $R_{11}^{*}+2 * R_{21}^{*}$ \\
\hline 7 & $\left\{1111 R_{11}^{*} P_{11}^{*}, 1133 R_{11}^{*} P_{11}^{*}, 2122 R_{21}^{*} P_{21}^{*}\right\}$ & $2 * R_{11}^{*}+R_{21}^{*}$ \\
\hline 8 & $\left\{2122 R_{21}^{*} P_{21}^{*}, 2133 R_{21}^{*} P_{21}^{*}, 1111 R_{11}^{*} P_{11}^{*}\right\}$ & $2 * R_{21}^{*}+R_{11}^{*}$ \\
\hline 9 & $\left\{2111 R_{21}^{*} P_{21}^{*}, 2122 R_{21}^{*} P_{21}^{*}, 1133 R_{11}^{*} P_{11}^{*}\right\}$ & $2 * R_{21}^{*}+R_{11}^{*}$ \\
\hline 10 & $\left\{2111 R_{21}^{*} P_{21}^{*}, 2122 R_{21}^{*} P_{21}^{*}, 2133 R_{21}^{*} P_{21}^{*}\right\}$ & $3 * R_{21}^{*}$ \\
\hline 11 & $\left\{1111 R_{11}^{*} P_{11}^{*}, 2122 R_{21}^{*} P_{21}^{*}\right\}$ & $R_{11}^{*}+R_{21}^{*}$ \\
\hline 12 & $\left\{1133 R_{11}^{*} P_{11}^{*}, 2122 R_{21}^{*} P_{21}^{*}\right\}$ & $R_{11}^{*}+R_{21}^{*}$ \\
\hline 13 & $\left\{1122 R_{11}^{*} P_{11}^{*}, 2111 R_{21}^{*} P_{21}^{*}\right\}$ & $R_{11}^{*}+R_{21}^{*}$ \\
\hline 14 & $\left\{1111 R_{11}^{*} P_{11}^{*}, 2133 R_{21}^{*} P_{21}^{*}\right\}$ & $R_{11}^{*}+R_{21}^{*}$ \\
\hline 15 & $\left\{1122 R_{11}^{*} P_{11}^{*}, 2133 R_{21}^{*} P_{21}^{*}\right\}$ & $R_{11}^{*}+R_{21}^{*}$ \\
\hline 16 & $\left\{1133 R_{11}^{*} P_{11}^{*}, 2111 R_{21}^{*} P_{21}^{*}\right\}$ & $R_{11}^{*}+R_{21}^{*}$ \\
\hline
\end{tabular}

of finding the maximum weight clique. These phases are explained as follows.

Phase I: CRAN-CLNC construction complexity: The computational complexity of constructing the CRAN-CLNC graph is divided into the following steps: i) generating a single subgraph for each $R R B$, ii) computing the power levels of the RRHs, and iii) connecting all the subgraphs in the system to construct the CRAN-CLNC graph. These steps are explained as follows.

First step: The complexity of this step corresponds to the computational complexity of generating all vertices in any subgraph. Consider computing the complexity of subgraph $\mathcal{G}_{z_{m}}$ of the $z_{m}$-th RRB. As explained in Section 3.2, the total number of vertices in each subgraph is the total number of possible feasible schedules $\left|\mathcal{S}_{z_{m}, \mathrm{fs}}\right|$. Each schedule $\mathbf{S} \in \mathcal{S}_{z_{m}, \mathrm{fs}}$ contains many possible associations that represent IDNC file combinations to be transmitted to a set of users from a set of RRHs with their corresponding transmission rates/powers. $\left|\mathcal{S}_{z_{m}, \mathrm{fs}}\right|$ depends on all possible IDNC file combinations $\mathcal{S}_{\text {IDNC }}$ in the system. Any possible IDNC combination contains combined files correspond to associated requesting users. We consider the worst-case complexity of generating the IDNC combinations as follows. First, note that each associated user with any IDNC file combination can receive at most one file. In other words, each user can instantly decode at most one file from its associated IDNC combination. Therefore, the maximum number of associations in any IDNC combination is $\min (U, F)$. In our setting, the number of files is greater than the number of users ${ }^{1}$. Thus, the upper bound of the IDNC combinations is $\left(\begin{array}{l}F \\ U\end{array}\right)$, which can be computed as $\frac{F !}{(F-U) ! U !}$. For simplicity, it can be denoted by $C=\frac{F !}{(F-U) ! U !}$. Second, note that $\mathcal{G}_{z_{m}}$ corresponds to the $z_{m}$-th RRB across all RRHs. Since all RRHs cache the whole set of files, each RRH can generate the same set of IDNC combinations that can be generated by the other RRHs. Further, the same IDNC combination and its corresponding users can not be associated to different RRHs in the same schedule $\mathbf{S}$ as this violates the condition LC2 that explained in Section 3.2. Hence, the possible IDNC combinations for all RRHs in the system can be permuted between all RRHs, and each permutation represents schedule $\mathbf{S}$. As previously mentioned, schedule $\mathbf{S}$ is represented by vertex $v$ in $\mathcal{G}_{z_{m}}$. Therefore, the total number of vertices in $\mathcal{G}_{z_{m}}$ is the number of permutations of these IDNC combinations among $B$ RRHs, which is $\frac{C !}{(C-B) !}$.

Second step: To compute the power levels, we solve (8) for each vertex $v$ in each subgraph. Solving (8) for each $v$ depends on the number of RRHs, number of users in $\mathrm{S}$, and interference pricing messages from the RRHs. The number of users in each schedule $\mathbf{S}$ is upper bounded by $U$, i.e., the case when all users' files can be combined using NC. For the worst-case complexity of computing (8), Algorithm 1 needs for each scheduled user in RRH $b_{n}$ to compute the interference of all other RRHs, i.e., $b_{n^{\prime}} \neq b_{n}$ and the pricing messages from each RRH $b_{\tilde{n}} \neq b_{n^{\prime}}$. Thus, the computational complexity of calculating the power allocation is upper bounded by $O(U(B(B-1)+B(B-1)))=$ $O\left(2 U\left(B^{2}-B\right)\right)=O\left(2 U B^{2}\right)$.

Third step: The number of subgraphs is the number of RRBs in the system. Thus, $Z$ subgraphs constitutes the CRAN-CLNC graph, and it has $\frac{C !}{(C-B) !} Z$ vertices. Therefore, the computational complexity of constructing the CRAN-CLNC graph and computing the power allocations is $O\left(\frac{C !}{(C-B) !} Z\left(2 U B^{2}\right)\right)$.

Phase II: Maximum-weight search complexity: First, we build the adjacency matrix of the CRAN-CLNC graph. Particularly, we need to check the General Condition (GC) that explained in Section 3.3 of each pair of $\frac{C !}{(C-B) !} Z$ vertices to determine whether they should be connected by an edge. This adjacent connection between vertices needs a complex-

1. If we increase the number of files without changing the number of users and RRHs, the complexity of the whole system will decrease. This is because for a large number of files, any set of randomly picked uncoded files by the RRHs has a high chance to satisfy all users. Thus, no need to perform network coding in this scenario. 
ity of $O\left(\left(\frac{C !}{(C-B) !} Z\right)^{2}\right)$. The computational complexity of the maximum-weight search algorithm can be decomposed in sum weights and vertex search computations as follows. Each iteration requires a complexity of $O\left(\frac{C !}{(C-B) !} Z\right)$ for weight calculations of its maximum-weight clique. Note that each maximum-weight clique has at most $Z$ vertices as each subgraph can contribute at most with one vertex per transmission. Therefore, building the adjacency matrix and executing the maximum-weight search have a complexity of $O\left(\left(\frac{C !}{(C-B) !} Z\right)^{2}+\frac{C !}{(C-B) !} Z+Z\right)=O\left(\left(\frac{C !}{(C-B) !} Z\right)^{2}\right)$. Given the aforementioned computational complexities of the different algorithm components, the total per-iteration computational complexity of Algorithm 1 in terms of main system's parameters is upper bounded by

$O\left(\frac{\left(\frac{F !}{(F-U) ! U !}\right) !}{\left(\frac{F !}{(F-U) ! U !}-B\right) !} Z\left(2 U B^{2}\right)+\left(\frac{\left(\frac{F !}{(F-U) ! U !}\right) !}{\left(\frac{F !}{(F-U) ! U !}-B\right) !} Z\right)^{2}\right)$. Since the second term dominates the overall complexity, we can write the upper bound overall complexity as $O\left(\left(\frac{\left(\frac{F !}{(F-U) ! U !}\right) !}{\left(\frac{F !}{(F-U) ! U !}-B\right) !} Z\right)^{2}\right)$. In general, the computational complexity of the joint solution is less than that as not all files can be combined due to the instant decodability conditions that explained in Section 2.2. In the uncoded case [30], the computational complexity is $O\left(\frac{U !}{(U-B) !} Z\left(2 B^{3}\right)+\left(\frac{U !}{(U-B) !} Z\right)^{2}\right)$.

For the high implementation complexity of the joint solution in large networks, we develop in the next section an efficient and alternative coordinated scheduling solution that has low implementation complexity.

\section{Iterative Optimization for CoORdinated Scheduling AND POWER Control}

We present in this section an efficient and low-complexity iterative solution to the optimization problem (5). In particular, we solve the coordinated NC schedule and power control separately and iteratively upon convergence.

We fix the power levels PLs in (5) and solve the following simplified problem to refine NC and user-RRB/RRH schedule

$$
\begin{aligned}
& \max \sum_{b_{n} \in \mathcal{B}} \sum_{z_{m} \in \mathcal{Z}} \sum_{u_{i} \in \mathcal{U}} X_{b_{n} z_{m}}^{u_{i}} \min _{u_{i^{\prime}} \in \tau_{b_{n^{\prime}} z_{m^{\prime}}}} R_{b_{n^{\prime}} z_{m^{\prime}}}^{u_{i^{\prime}}} \\
& \text { s.t. }(5 b),(5 c),(5 e) .
\end{aligned}
$$

The optimization is carried over the variables $X_{b_{n} z_{m}}^{u_{i}}$ $Y_{b_{n}}^{u_{i}}$, and $\kappa_{b_{n} z_{m}}$.

Then, we use the resulting NC and user-RRB/RRH schedule in (5) and solve the following problem per RRBs basis

$$
\begin{aligned}
& \max _{P_{b_{n} z_{m}}} \sum_{b_{n} \in \mathcal{B}}\left|\tau_{b_{n} z_{m}}\right| * \min _{u_{i} \in \tau_{b_{n} z_{m}}} \log _{2}\left(1+\operatorname{SINR}_{b_{n} z_{m}}^{u_{i}}(\mathbf{P})\right) \\
& \text { s.t. } 0 \leqslant P_{b_{n} z_{m}} \leqslant P_{b_{n} z_{m}}^{\max }, \forall b_{n} \in \mathcal{B}
\end{aligned}
$$

where $P_{b_{n}} z_{m}, \forall b \in \mathcal{B}$ is the set of optimization parameters, $\mathcal{B}$ is the set of RRHs, and $u_{i}$ is the user that belongs to the set of targeted users $\tau_{b_{n} z_{m}}$ that have associations in the resulting schedule. In what follows, we show how to solve problems (9) and (10), respectively.

\subsection{Coordinated Scheduling: Solution to Problem (9)}

Let $\mathcal{S}$ be the set of all possible associations (all pairs) between RRHs, RRBs, users, files and the achievable capacity, i.e., $\mathcal{S}=\mathcal{B} \times \mathcal{Z} \times \mathcal{U} \times \mathcal{F} \times \mathcal{R}$, and $s$ is an element in $\mathcal{S}$. For example, $s=\left(b_{n}, z_{m}, u_{i}, f_{k}, R\right) \in \mathcal{S}$ represents the RRH $b_{n}, \operatorname{RRB} z_{m}$, user $u_{i}$, file $f_{k}$, and rate $R$. Let the coordinated scheduling graph be denoted by $\mathcal{G}(\mathcal{V}, \mathcal{E})$ wherein $\mathcal{V}$ and $\mathcal{E}$ refer to the set of vertices and edges of this graph, respectively. This graph is constructed by generating a vertex $v$ for each possible association $s \in \mathcal{S}$.

In the same $z_{m}$-th RRB and $b_{n}$-th RRH, two vertices $v \in \mathcal{V}$ associated with $s \in \mathcal{S}$ and $v^{\prime} \in \mathcal{V}$ associated with $s^{\prime} \in \mathcal{S}$ are connected by an edge if one of IDNC conditions (C1 or C2) in Section 3.2 and $R(s)=R\left(s^{\prime}\right)$ are satisfied. This satisfaction ensures that all users represented by the associations have the same transmission rate, and receive always decodable transmission.

Similarly, two different vertices belonging to two different RRHs/RRBs are then set adjacent by an edge if their combination results in a feasible schedule. Let vertex $v \in \mathcal{G}$ be corresponding to the association $s \in \mathcal{S}$ and vertex $v^{\prime} \in \mathcal{G}$ corresponding to the association $s^{\prime} \in \mathcal{S}$. The vertices $v, v^{\prime}$ are adjacent if one of the general conditions (GC) is satisfied.

- GC1: $u_{i}(s)=u_{i}\left(s^{\prime}\right)$ and $b_{n}(s)=b_{n}\left(s^{\prime}\right), \forall\left(s, s^{\prime}\right) \in \mathcal{S}$. This condition represents that the same user can be served from multiple RRBs within the same RRH.

- GC2: $\quad\left(b_{n}(s)=b_{n}\left(s^{\prime}\right)\right.$ and $\left.f_{k}(s)=f_{k}\left(s^{\prime}\right)\right) \quad$ OR $\left(b_{n}(s)=b_{n}\left(s^{\prime}\right)\right.$ and $f_{k}(s) \in \mathcal{H}_{u_{i}\left(s^{\prime}\right)}$ and $\left.f_{k}\left(s^{\prime}\right) \in \mathcal{H}_{u_{i}(s)}\right)$. This condition guarantees that the encoded combinations of the same users can be served by multiple RRBs within the same RRH.

- GC3: $u_{i}(s) \neq u_{i}\left(s^{\prime}\right)$ and $\left(b_{n}(s), z_{m}(s)\right) \neq$ $\left(b_{n}\left(s^{\prime}\right), z_{m}\left(s^{\prime}\right)\right)$. This condition completes the adjacencies in the graph for any two vertices not opposing the $\mathbf{C C} 1$ constraint for any two different users.

Example 2: We show in this example the coordinated scheduling graph and its corresponding cliques of the network presented in Example 1. In Fig. 5, each vertex $v$ is labeled by the subscripts $n m i k R$ of the association $s=\left(b_{n}, z_{m}, u_{i}, f_{k}, R\right)$, where $n, m, i, k$ and $R$ represent the indices of RRHs, RRBs, users, files, and rates, respectively. The dashed lines in Fig. 5 represent either $\mathrm{NC}$ conditions $(\mathbf{C} 1, \mathbf{C} 2)$ or rate condition $\left(R(s)=R\left(s^{\prime}\right)\right)$, and the solid lines represent GC1. There are many possible maximal cliques in $\mathcal{G}$ that are represented by connected vertices. Each clique represents the potential network-coded scheduling of the RRHs/RRBs that maximizes throughput. For example, the potential cliques in this example represented in Fig. 5 by solid lines are: $\{11111,21221\},\{21332,11111\}, \quad\{21221,11332\}$, \{21111,21221, 21331\}, $\quad\{11111,11221,11331\}$, $\{21112,11222,11332\}$, and $\{11223,21112,21332\}$. The achieving total throughputs of these cliques are: $2,3,3,3$, 3,6 , and 7 bits/s, respectively. Clearly, the vertices (circles) connected by red solid lines represent the last maximal clique, and should be the one selected as it offers the maximum throughput. It can be noted that any clique in Fig. 5 satisfies the instant decodablity transmission to all users have vertices in it. 


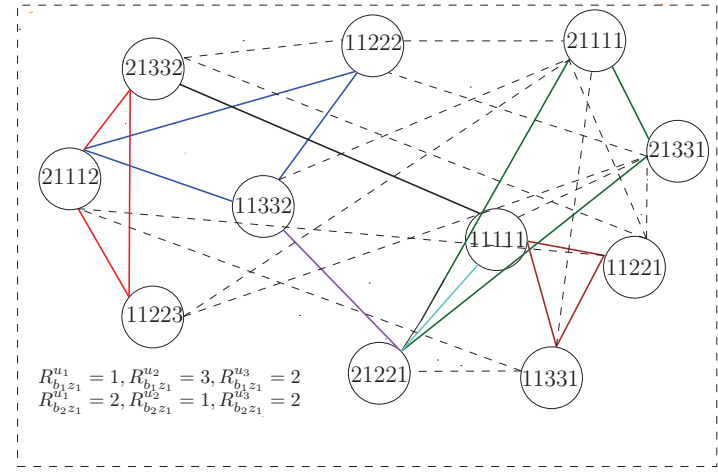

Fig. 5. The coordinated scheduling graph of the network presented in Fig. 3 using Algorithm 2.

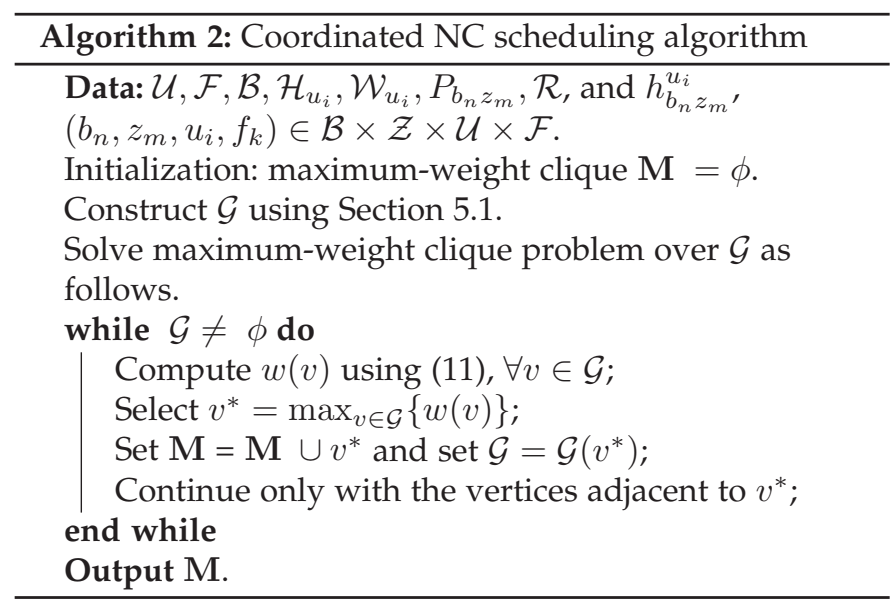

The following theorem characterizes the solution to the coordinated scheduling problem (9).

Theorem 2. The coordinated scheduling problem in (9) is equivalent to a maximum-weight clique problem over the coordinated scheduling graph, wherein the weight of a vertex $v \in \mathcal{V}$ corresponding to the association $s=\left(b_{n}, z_{m}, u_{i}, f_{k}, R\right) \in \mathcal{S}$ is given by

$$
w(v)=R .
$$

Problem (11) is NP-hard [48]. Therefor, we make use one of the exiting algorithms, e.g., [45]-[47], to solve (11) heuristically in linear time with respect to its size as shown in Algorithm 2.

\subsection{Power Allocation: Solution to Problem (10)}

Given the resulting NC and user-RRB/RRH schedule in problem (10), finding the power level PL of each RRB is a non-convex problem. Thus, we focus on a numerical solution to achieve at least a local optimum solution using the Karush-Kuhn-Tucker (KKT) iteration approach. In particular, the corresponding power levels for the resulting $\mathrm{NC}$ and user-RRB/RRH schedule must satisfy the first derivative, i.e., KKT condition. Therefore, the objective function of the problem (10) which is optimized over the set of powers on an RRB-by-RRB basis, can be expressed as

$$
\begin{aligned}
& R\left(P_{b_{1} z_{m}}, P_{b_{2} z_{m}}, \ldots, P_{b_{B} z_{m}}\right)= \\
& \min _{u_{i} \in \tau_{b_{n} z_{m}}} \sum_{b_{n} \in \mathcal{B}} \sum_{u_{i} \in \tau_{b_{n} z_{m}}} \log _{2}\left(1+\frac{P_{b_{n} z_{m}}\left|h_{b_{n} z_{m}}^{u_{i}}\right|^{2}}{\sigma^{2}+\sum_{b_{n^{\prime}} \neq b_{n}} P_{b_{n^{\prime}} z_{m}}\left|h_{b_{n^{\prime}} z_{m}}^{u_{i}}\right|^{2}}\right) \\
& \text { s.t. } 0 \leqslant P_{b_{n} z_{m}} \leqslant P_{b_{n} z_{m}}^{\max }, \forall b_{n} \in \mathcal{B} .
\end{aligned}
$$

The steps of solving the power optimization problem in (10) is given as follows.

First, we take the first derivative of the objective function (14a) with respect to $P_{b_{n} z_{m}}$ as in (12) and (13) at the top of the next page.

Second, we let the gradient in (12) equal to zero. Then, by manipulating the optimality condition, we obtain this manipulation for optimizing the power

$$
\begin{aligned}
& \frac{1}{P_{b_{n} z_{m}}} \sum_{u_{i} \in \tau_{b_{n} z_{m}}}\left(\frac{\operatorname{SINR}_{b_{n} z_{m}}^{u_{i}}}{1+\operatorname{SINR}_{b_{n} z_{m}}^{u_{i}}}\right) \\
& =\sum_{b_{n^{\prime}} \neq b_{n}} \sum_{u_{i^{\prime}} \in \tau_{b_{n^{\prime}} z_{m}}} \frac{\left|h_{b_{n} z_{m}}^{u_{i^{\prime}}}\right|^{2}}{P_{b_{n^{\prime}} z_{m}}\left|h_{b_{n^{\prime}} z_{m}}^{u_{i^{\prime}}}\right|^{2}}\left(\frac{\left(\mathrm{SINR}_{b_{n^{\prime}} z_{m}}^{u_{i^{\prime}}}\right)^{2}}{1+\operatorname{SINR}_{b_{n^{\prime}} z_{m}}^{u_{i^{\prime}}}}\right)
\end{aligned}
$$

Therefore,

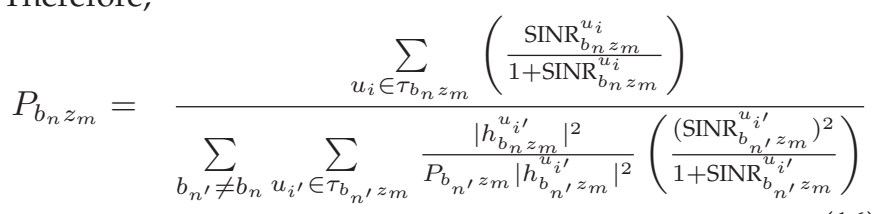

Finally, we compute the optimal $\left[P_{b_{n} z_{m}}\right]_{n=1}^{B}$ by interpreting the power condition iteratively. More precisely, given the power allocation at the first iteration, the right-hand side of (16) can be computed using the current power, then the new results are used to update the new power allocations. Furthermore, to account for the RRB power constraint, an additional projection on $[.]_{0}^{P^{\max }}$ can be taken such that in each iteration, the power level $P_{b_{n} z_{m}}$ is updated according to the following

$$
P_{b_{n} z_{m}, \text { new }}=\left[\frac{\sum_{u_{i} \in \tau_{b_{n} z_{m}}}\left(\frac{\operatorname{SINR}_{b_{n} z_{m}}^{u_{i}}}{1+\operatorname{SINR}_{b_{n} z_{m}}^{u_{i}}}\right)}{\sum_{b_{n^{\prime}} \neq b_{n}} t_{b_{n^{\prime}} z_{m}}}\right]_{0}^{P^{\max }},
$$

where,

$$
t_{b_{n^{\prime}} z_{m}}=\sum_{u_{i^{\prime}} \in \tau_{b_{n^{\prime}} z_{m}}} \frac{\left|h_{b_{n} z_{m}}^{u_{i^{\prime}}}\right|^{2}}{P_{b_{n^{\prime}} z_{m}}\left|h_{b_{n^{\prime}} z_{m}}^{u_{i^{\prime}}}\right|^{2}}\left(\frac{\left(\operatorname{SINR}_{b_{n^{\prime}} z_{m}}^{u_{i^{\prime}}}\right)^{2}}{1+\operatorname{SINR}_{b_{n^{\prime}} z_{m}}^{u_{i^{\prime}}}}\right)
$$

and the notation $[a]_{y}^{x}$ represents $a$ upper bounded above by $x$ and lower bounded below by $y$. Note that the iterative process of (17) must converge to a point either lie in the interior of the constrained set or on the boundary, so as to satisfy the "KKT condition" of the optimization problem (for details see [20], [43], [49]). It is important to note that, the nominator on the right-hand side of (17) represents the effect power of $z_{m}$-th RRB in $b_{n}$-th RRH on all corresponding RRHs in the schedule, i.e., it is the derivative of the $b_{n^{\prime}}$ th RRHs terms with respect to the $z_{m}$-th RRB power in the $b$ th RRH. In other words, it summarizes the interfering effect of $P_{b_{n} z_{m}}$ on the $b_{n^{\prime}}$ th RRH. Moreover, it depends on the transmit power, SINR, and the ratio of the direct and the interfering channel gains. The dominator on the righthand side of (17) shows the effect of the combined noise and interference in $z_{m}$-th RRB of $b_{n}$-th RRH.

The overall two-step algorithm to problem (5) is summarized in Algorithm 3. 


$$
\begin{aligned}
& \frac{\partial R}{\partial P_{b_{n} z_{m}}}=\frac{\partial}{\partial P_{b_{n} z_{m}}} \sum_{u_{i} \in \tau_{b_{n} z_{m}}} \log _{2}\left(1+\frac{P_{b_{n}}^{\text {Transactions on Mobile Computing }}}{\sigma^{2}+\left.\sum_{b_{n^{\prime}} \neq b_{m}} P_{b_{n}}^{u_{n} z_{m}}\right|_{n_{m}}\left|h_{b_{n^{\prime}} z_{m}}^{u_{i}}\right|^{2}}\right) \\
& +\frac{\partial}{\partial P_{b_{n} z_{m}}} \sum_{b_{n^{\prime}} \neq b_{n}} \sum_{u_{i^{\prime}} \in \tau_{b_{n^{\prime}} z_{m}}} \log _{2}\left(1+\frac{P_{b_{n^{\prime}} z_{m}}\left|h_{b_{n^{\prime}} z_{m}}^{u_{i^{\prime}}}\right|^{2}}{\sigma^{2}+\sum_{b_{\tilde{n}} \neq b_{n^{\prime}}} P_{b_{\tilde{n}} z_{m}}\left|h_{b_{\tilde{n}} z_{m}}^{u_{i^{\prime}}}\right|^{2}}\right)=\frac{1}{P_{b_{n} z_{m}}} \sum_{u_{i} \in \tau_{b_{n} z_{m}}}\left(\frac{\operatorname{SINR}_{b_{n} z_{m}}^{u_{i}}}{1+\operatorname{SINR}_{b_{n} z_{m}}^{u_{i}}}\right) \\
& -\sum_{b_{n^{\prime}} \neq b_{n}} \sum_{u_{i^{\prime}} \in \tau_{b_{n^{\prime}}} z_{m}} \frac{\left|h_{b_{n} z_{m}}^{u_{i^{\prime}}}\right|^{2}}{P_{b_{n^{\prime}} z_{m}}\left|h_{b_{n^{\prime}} z_{m}}^{u_{i^{\prime}}}\right|^{2}}\left(\frac{\left(\operatorname{SINR}_{b_{n^{\prime}} z_{m}}^{u_{i^{\prime}}}\right)^{2}}{1+\operatorname{SINR}_{b_{n^{\prime}}}^{u_{i_{m}}}}\right)
\end{aligned}
$$

where,

$$
\operatorname{SINR}_{b_{n^{\prime}} z_{m}}^{u_{i^{\prime}}}=\left(1+\frac{P_{b_{b^{\prime}} z_{m}}\left|h_{b_{n^{\prime}} z_{m}}^{u_{i^{\prime}}}\right|^{2}}{\sigma^{2}+\sum_{b_{\tilde{n}} \neq b_{n^{\prime}}} P_{b_{\tilde{n}} z_{m}}\left|h_{b_{\tilde{n}} z_{m}}^{u_{i^{\prime}}}\right|^{2}}\right)
$$

and $u_{i} \in \tau_{b_{n} z_{m}}$ and $u_{i^{\prime}} \in \tau_{b_{n^{\prime}} z_{m}}$ are the scheduled users of the $b_{n}$-th RRH, and the $b_{n^{\prime}}$ th RRH at the $z_{m}$ th RRB for $\forall b_{n}, b_{n^{\prime}}$ $\in \mathcal{B}$, respectively.

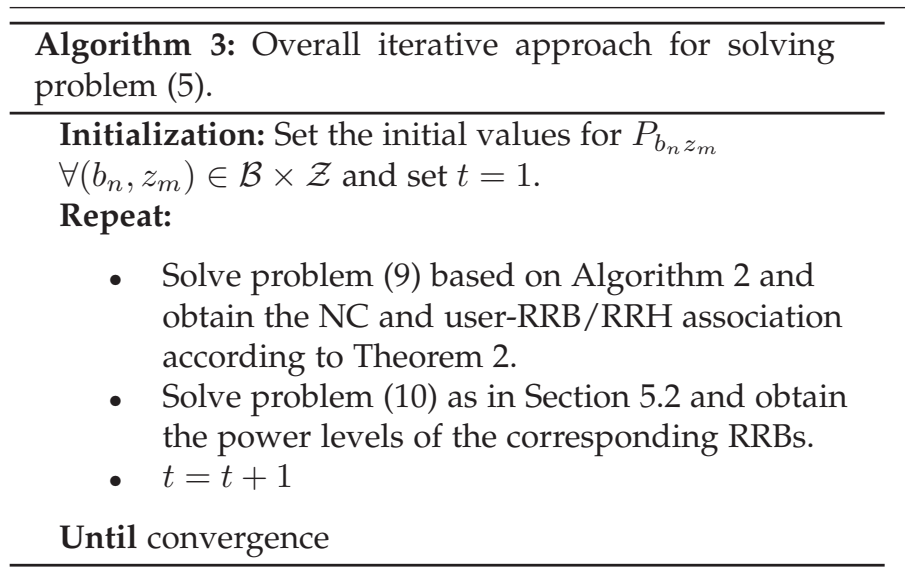

\subsection{Complexity of Coordinated Scheduling Heuristic Solution}

In this subsection, we compute the complexity of constructing the coordinated scheduling graph, i.e., generating the vertices and connecting them, and executing the maximumweight search. These steps are explained as follows.

First step: We construct the coordinated scheduling graph by generating $O(U F|\mathcal{R}|)$ vertices, where $|\mathcal{R}|=U Z$. These vertices represent the requested popular files by users, which can be targeted by one RRB with one of the achievable rates. However, vertices representing different rates cannot be part of one clique in each RRB in the same RRH. Clearly, avoiding such vertices reduces the complexity to $O(U F)$. Generalizing this to the whole system has a complexity of $O(U F Z B)$. Then, we iteratively search over these vertices for each rate as will be explained later. The adjacency matrix that connects vertices by edges needs a complexity of $\mathcal{O}\left(U^{2} F^{2} Z B\right)$ for coding conditions, and $O\left(U^{2} F^{2} Z^{2} B^{2}\right)$ for general conditions. Thus, the complexity of building the adjacency matrix is $O\left(U^{2} F^{2} Z B+U^{2} F^{2} Z^{2} B^{2}\right)=O\left(U^{2} F^{2} Z^{2} B^{2}\right)$.

Second step: The maximum-weight search algorithm iteratively search over all vertices for each rate of each RRB in each RRH. This needs at most $|\mathcal{R}|$ maximal cliques. Since each user cannot be scheduled to more than one RRH, each maximal clique has at most $U$ vertices. Further, each user can be targeted by at most one file (i.e., one vertex) from one RRB across all RRHs. Thus, the calculation of the weights in each maximal clique and its number of vertices (considering the lowest rate that involves all users ) has a complexity of $O(B|\mathcal{R}|[(U F+U)])$. In the whole system, the complexity is
$O(B Z|\mathcal{R}|[(U F+U)])$.

Third step: We combine all the complexities of the different algorithm elements that previously explained, which yields the total complexity of the algorithm per iteration of $O\left(U F Z B+U^{2} F^{2} Z^{2} B^{2}+B Z|\mathcal{R}|[(U F+U)]\right)$. Overall, roughly, the second term dominates the total complexity as $|\mathcal{R}| \leqslant U Z$, which abstracts the complexity of Algorithm 2 to $O\left(U^{2} F^{2} Z^{2} B^{2}\right)$.

Communication and storage overheads: It is important to recall that the whole process in this paper is done by the $\mathrm{CP}$. Furthermore, the $\mathrm{CP}$ knows users' side information and stores them in a log ledger. Therefore, compared to the standard uncoded solution, coded scheme needs nothing. However, the coding scheme may need overhead for the headers of each encoded transmission (to notify users of the XORed files in each transmission), but this will not exceed a few bytes. It is also worth noting that the encoded file has the same size as the source files, which simplifies both the coding overhead at the RRBs/RRHs and the users' decoding complexity [36]. On the other hand, for storage overheads, users need to cache some files to be used later as side information. Such cached file is refreshed over a short period of time so as it does not increase with time, i.e., newer popular files are replacing older non-used files. Furthermore, increasing the capabilities of devices as well as reducing their memory costs enable that easily. Thus, as long as the number of cached files is bounded, i.e., window size in our case, the cached files would not consider as overheads (for more details about caching, see for example [50]).

\section{Numerical Results}

We consider a downlink C-RAN system that described in Section 2 and plotted in Fig. 1. The system setting in this manuscript follows the setup studied in [30] and [39]. The number of RRHs is fixed to 3 unless stated. The additional numerical parameters are summarized in Table 5.

Each presented value in each plot for the joint and the iterative schemes is obtained by averaging the throughput expressions in Theorem 1 and Theorem 2 over a large number of iterations, respectively. At each run, users' side information is randomly generated. To assess the performances of our proposed schemes, we simulate various scenarios: number of users $U$, number of RRBs per each RRH's frame, maximum power $P^{\max }$, cell size $D$, and file sizes.

For numerical comparison, the proposed solutions are compared with the following schemes. 
TABLE 5

Numerical parameters

\begin{tabular}{|l|l|}
\hline Bandwidth & $10 \mathrm{MHz}$ \\
\hline Cellular layout & Hexagonal \\
\hline Channel estimation & Perfect \\
\hline Channel model & SUI-Terrain type B \\
\hline Path loss model & $128.1+37.6 \log _{10}($ dis.[km] $)$ \\
\hline Background noise power & $-168.60 \mathrm{dBm} / \mathrm{Hz}$ \\
\hline Shadowing variance & $0 \mathrm{~dB}$ \\
\hline Distribution of users & Uniform \\
\hline
\end{tabular}

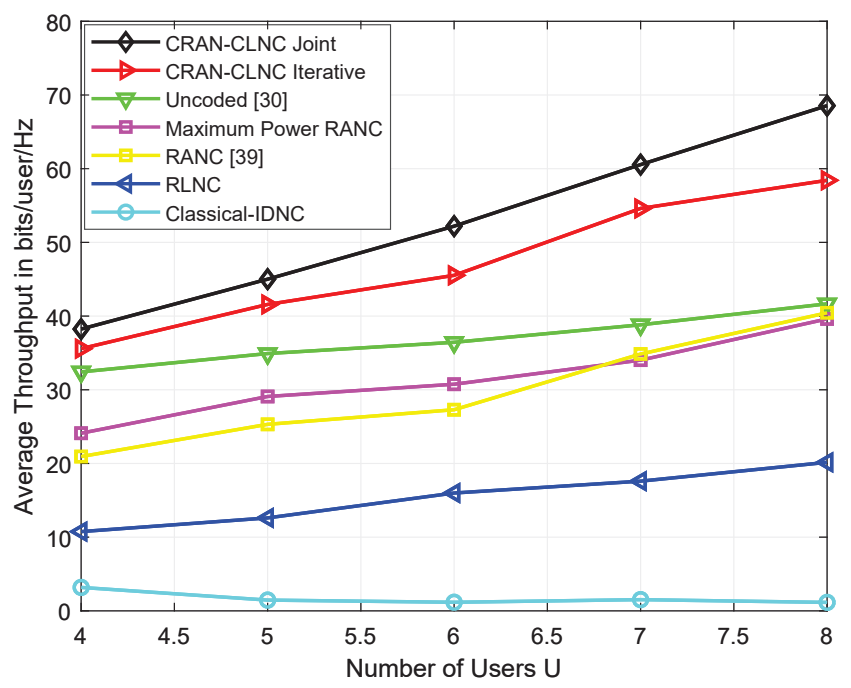

Fig. 6. Average throughput in bits/user/Hz. vs the number of users $U$.

- Classical IDNC: This scheme optimizes the selection of XOR file combinations with the absence in physical layer aspects. For full file's reception, the RRB's transmission rate is limited by the weakest user who experiences the lowest transmission capacity.

- RLNC: This scheme associates users to a single RRB to which it has the maximum capacity. The random linear network coding (RLNC) is employed for each RRB that has multiple associated users. The encoding is done by mixing all files with different random coefficients, and the selected transmission rate in each RRB is limited by the minimum achievable capacity of the assigned users.

- Maximum power RANC: This scheme uses RANC code while assuming the transmission power of all RRBs is $P^{\max }$.

- Uncoded: This scheme was studied in [30] where only physical-layer aspects were considered.

- RANC: This scheme was studied in [39].

In Fig. 6, we plot the average throughput versus the number of users $U$ for a C-RAN composed of 2 RRBs per RRH's frame, $F=10$ files, a file size of $1 \mathrm{Mb}$, a maximum transmit power $P^{\max }=-42.60 \mathrm{dBm}$, and a cell size $D=$ $500 \mathrm{~m}$. From the figure, we see that the performance gains of the proposed joint and iterative schemes outperform the performances of the other schemes in terms of throughput maximization. In particular, when $U$ is sufficiently small $(U=4,5)$, the uncoded scheme works closely to the proposed schemes, i.e., joint CRAN-CLNC scheme offers $28 \%$

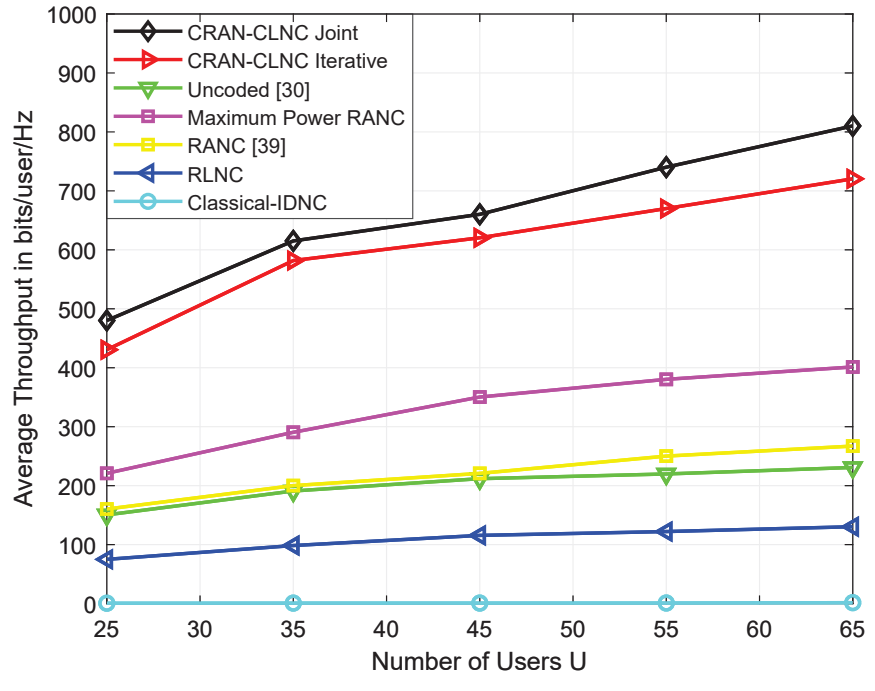

Fig. 7. Average throughput in bits/user/Hz. vs a large number of users $U$.

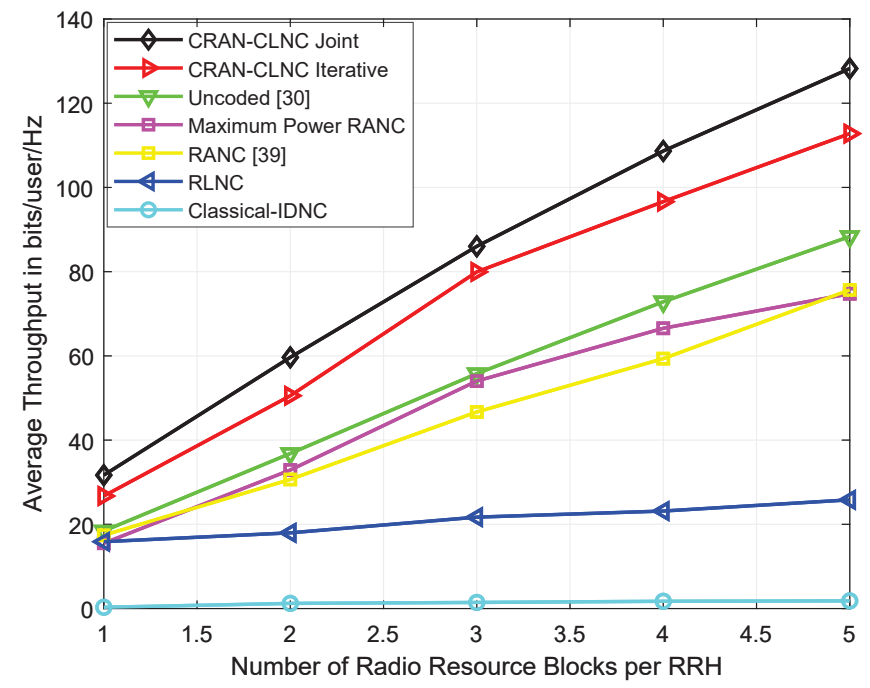

Fig. 8. Average throughput in bits/user/Hz. vs. the number of radio resource blocks $Z$.

improvement over uncoded for $U=5$. This is due to the fact that the proposed CRAN-CLNC schemes suffer from a small number of encoded scheduling opportunities in each $\mathrm{RRB} / \mathrm{RRH}$. In contrast, when $U$ is moderate $(U=7,8)$, the proposed joint scheme achieves an appreciable performance improvement ( $53 \%$ for $U=7$ and $63 \%$ for $U=8$ ) over the uncoded scheme. This is because the encoded opportunities of mixing users' flow in each RRB/RRH increases as the number of users $U$ increases.

In order to evaluate the performance of the proposed schemes in a large C-RAN, Fig. 7 illustrates average throughput versus a large number of users $U$ for a C-RAN composed of 6 RRHs, 5 RRBs per RRH's frame, and $F=68$ files, each with a size of $1 \mathrm{Mb}$. The figure suggests that, for the uncoded strategy, the average throughput increases as the number of users $U$ increases, but uncoded scheme serves only one user per RRB (serves in total $Z_{\text {tot }}$ users), which limits the overall gain in the average throughput. Conversely, if the number of users is large enough compared 


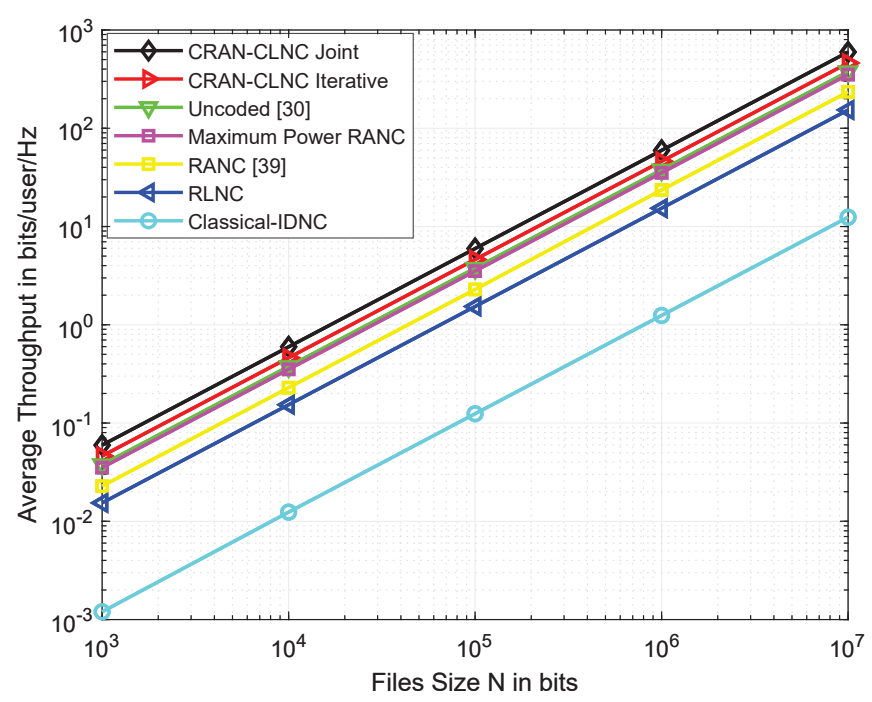

Fig. 9. Average throughput in bits/user/Hz. vs the file size $N$ in bits.

to $Z_{\text {tot, }}$ joint and iterative schemes achieve significant gains over the uncoded scheme (the average throughput is twice for $U=30$ and almost three times higher for $U=60$ ). We can also see that, with the limitation of rate equality for all RRHs, RANC scheme offers improved performance as compared to the uncoded scheme at sufficiently large $U$. Finally, the maximum power RANC scheme, which exploits the NC abilities, serves a good number of users in each transmission. Thus, it works better than the uncoded one in a large C-RAN system.

In Fig. 8, we investigate the impact of the number of RRBs $Z$ on the C-RAN's average throughput. We set the parameters $U=7, F=10, N=1 \mathrm{Mb}, P^{\max }=-42.60$ $\mathrm{dBm}$, and $D=500 \mathrm{~m}$. From this figure, it can be seen that the average throughput of all schemes increases as the number of RRBs $Z$ increases. This is because all schemes agree on serving the same set of users in different RRBs in the same RRH. However, the performances of the proposed schemes over the uncoded and the RANC schemes are pronounced. In fact, the proposed schemes emphasize the different relative behavior of the uncoded and RANC schemes. In particular, the uncoded scheme only focuses on the high achievable rates at the expense of transmitting at most one file to a single user from each RRB in all RRHs. The RANC scheme leverages the potential of rate-aware codes in maximizing the throughput, but it limits the transmission rate of the same RRB in all RRHs to the minimum, which affects the overall gain. Moreover, the proposed joint scheme offers improved performance as compared to the iterative one for larger $Z$ as the search space becomes larger for larger $Z$. This becomes more advantageous for the proposed CRAN-CLNC joint algorithm.

In Fig. 9, we depict the average throughput versus the file size $N$ in a C-RAN system composed of 7 users, 9 files, and 2 RRBs per RRH's transmit frame, each RRB has a maximum transmit power $P^{\max }=-42.60 \mathrm{dBm}$. The cell size $D$ is set to $500 \mathrm{~m}$. Note that average throughput of all schemes are increased linearly with the increase in the file size. In fact, as the size of the file increases, more bits are received, thus increasing the average received throughput.
In Fig. 10, we plot the average throughput versus the maximum power $P^{\max }$, for a C-RAN setting composed of 2 RRBs in each RRH's transmit frame, 7 users, 9 files, a file size $N=1 \mathrm{Mb}$, and a cell size $D=500 \mathrm{~m}$. We can see from the figure that the achieved throughput of the CRANCLNC joint scheme with a larger $P^{\max }$ is outperformed the iterative strategy. This is because, in the regime of large $P^{\max }$, i.e., high inter-RRHs interference, the iterative scheme does not consider the whole search space in the system and rather partial consideration. In contrast, the joint scheme as a method of power optimization considers the overall interference's effect in the system.

In Fig. 11, we show the average throughput versus the cell size $D$, for a C-RAN setting composed of 7 users, 9 files, 2 RRBs in each RRH's transmit frame, each RRB has a maximum transmit power $P^{\max }=-26.98 \mathrm{dBm}$, and a file size $N=1 \mathrm{Mb}$. The figure confirms the result in Fig. 10 that if the cell size is sufficiently small, i.e., high interference level, the proposed joint scheme can provide high gain in average throughput over the iterative one. As the cell size increases, i.e., low interference level, the performance of the proposed joint solution over the iterative one decreases and gains only $10 \%$ improvement.

Finally, we study the convergence behavior of the iterative approach that explained in Algorithm 3. We set the parameters $U=8, F=10, Z=2, P^{\max }=-42.60 \mathrm{dBm}$, $N=1 \mathrm{Mb}$, and cell size $D=500 \mathrm{~m}$. We plot the average throughput versus the number of iterations, as shown in Fig. 12. From this figure, it can be seen that solving the coordinated scheduling and the power allocation problems converges iteratively. This confirms the fact that both problems are increasing in the objective function.

It is worth remarking that classical IDNC scheme offers completion time reduction of a frame of files in a number of NC works. However, as mentioned before that serving many users in each RRB while limiting the transmission rate of the RRB to the minimum exhibits a poor performance from a cross-layer perspective. Therefore, by means of extensive numerical results, we showed in this work that CRAN-CLNC joint and iterative schemes provide a more effective way to use NC, power adaptation, and simultaneous transmissions in C-RAN setting than all other schemes. Our proposed iterative scheme has a certain degradation in throughput performance as compared to the proposed joint scheme. This small degradation in some numerical results, roughly in the range of $9 \%-14 \%$, comes at the achieved low computational complexity as compared to the high complexity of the joint scheme.

\section{Conclusion}

This paper introduced a cross-layer framework in downlink C-RAN to optimize throughput by streaming a set of popular files to users. For any arbitrary transmission, we considered optimizing the received throughput, subject to users' side information, the cached/stored files at each RRH, NC constraints, and the channel qualities. We proposed two schemes, namely, joint and iterative schemes. Specifically, in the joint scheme, we used a novel graph theoretical representation to solve the joint coordinated scheduling and power control problem, while in the iterative scheme, we 


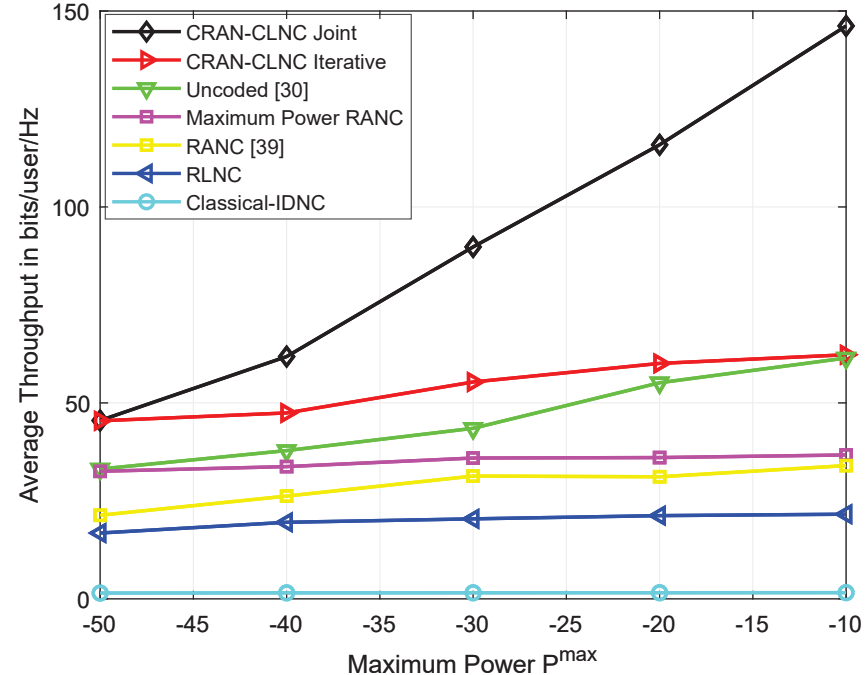

Fig. 10. Average throughput in bits/user/Hz. vs maximum power $P^{\max }$.

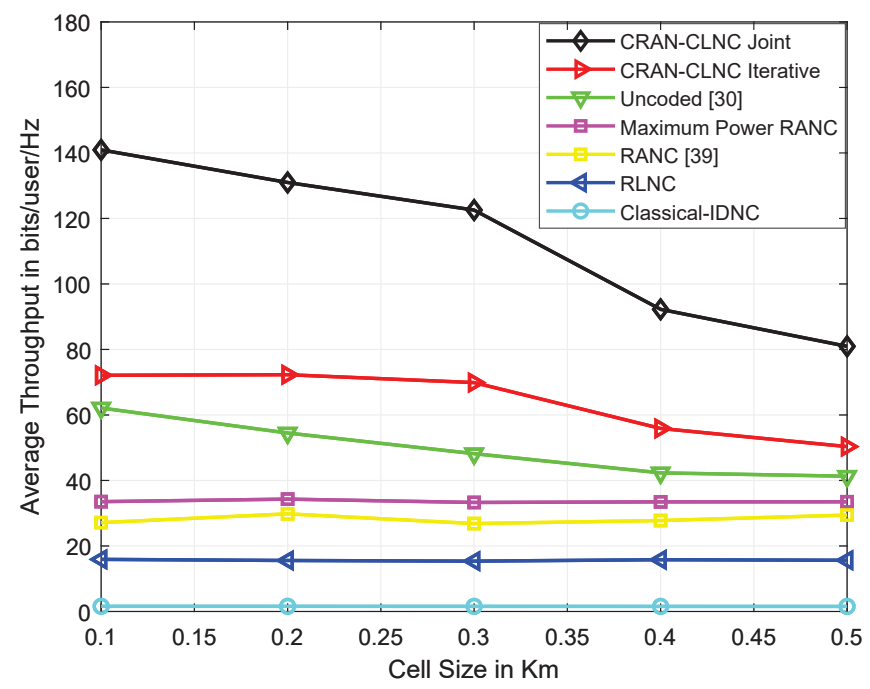

Fig. 11. Average throughput in bits/user/Hz. vs cell size $D$ in $K_{m}$.

solved the problem iteratively. Numerical results showed that both proposed schemes offer improved throughput performances as compared to the existing solutions.

\section{REFERENCES}

[1] M.-S. Al-Abiad and A. Douik and S. Sorour and Md. J. Hossain, "Throughput maximization in cloud radio access networks using network codes," in Proc. of 2018 IEEE Int. Conf. on Commun. Workshops (ICC Workshops), Kansas City, MO, 2018, pp. 1-6.

[2] Y. Cai, F.-R. Yu, and S. Bu, "Cloud computing meets mobile wireless communications in next generation cellular networks," IEEE Network, vol. 28, no. 6, pp. 54-59, Nov. 2014.

[3] O. Simeone, A. Maeder, M. Peng, O. Sahin, and W. Yu, "Cloud radio access network: Virtualizing wireless access for dense heterogeneous systems," J. Commun. Netw., vol. 18, no. 2, pp. 135-149, Apr. 2016.

[4] R. Zakhour and D. Gesbert, "Optimized data sharing in multicell MIMO with finite backhaul capacity," IEEE Trans. Signal Process., vol. 59, no. 12, pp. 6102-6111, Dec. 2011.

[5] B. Dai and W. Yu, "Sparse beamforming and user-centric clustering for downlink cloud radio access network," IEEE Access, vol. 2, pp. 1326-1339, Oct. 2014.

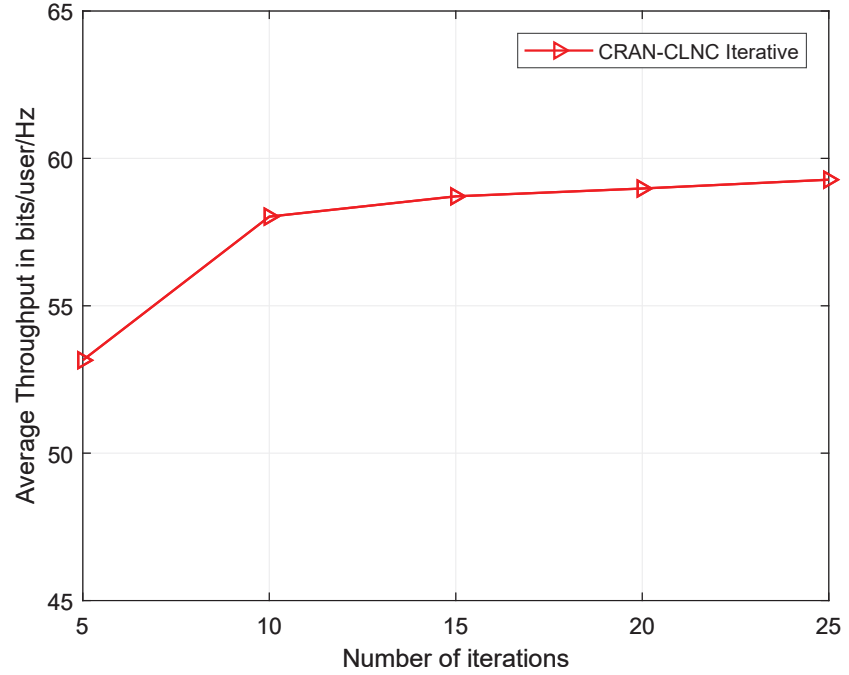

Fig. 12. Average throughput in bits/user/Hz. vs the number of iterations.

[6] S.-H. Park, O. Simeone, O. Sahin, and S. Shamai (Shitz), “Joint precoding and multivariate backhaul compression for the downlink of cloud radio access networks," IEEE Trans. Signal Process., vol. 61, no. 22, pp. 5646-5658, Nov. 2013.

[7] P. Patil and W. Yu. "Generalized compression strategy for the downlink cloud radio access network." [Online]. Available: https://arxiv.org/abs/1801.00394.

[8] S. Smirani, M. Kamoun, M. Sarkiss, A. Zaidi, and P. Duhamel, "Achievable rate regions for two-way relay channel using nested lattice coding," in IEEE Trans. on Wireless Commun., vol. 13, no. 10, pp. 5607-5620, Oct. 2014.

[9] I.-E. Aguerri and A. Zaidi, "Lossy compression for compute-andforward in limited backhaul uplink multicell processing," in IEEE Trans. on Commun., vol. 64, no. 12, pp. 5227-5238, Dec. 2016.

[10] N. Liu and W. Kang, "A new achievability scheme for downlink multicell processing with finite backhaul capacity," in Proc. IEEE Int. Symp. Inf. Theory (ISIT), Honolulu, HI, USA, Jun./Jul. 2014.

[11] C.-Yi Wang, M. Wigger, and A. Zaidi, "On achievability for downlink cloud radio access networks with base station cooperation," IEEE Trans. on Inf. Theory, vol. 64, no. 8, pp. 5726-5742, Aug. 2018.

[12] R. Ahlswede, N. Cai, S.-Y. Li, and R. Yeung, "Network information flow," IEEE Trans. on Inf. Theory, vol. 46, no. 4, pp. 1204-1216, Jul. 2000.

[13] L. Liu and W. Yu. "Cross-layer design for downlink multihop cloud radio access networks with network coding," IEEE Trans. Signal Process., vol. 65, no. 7, pp. 1728- 1740, Apr. 2017.

[14] Y. Wu, P.-A. Chou, Q. Zhang, K. Jain, W. Zhu, and S.Y. Kung, "Network planning in wireless Ad Hoc networks: a crosslayer approach," IEEE journal on Selected Areas in Commun., vol. 23, no. 1, pp. 136-150, Jan. 2005.

[15] Y. E. Sagduyu and A. Ephremides, “On joint MAC and network coding in wireless Ad Hoc networks," IEEE Trans. on Inf. Theory, vol. 53, no. 10, pp. 3697-3713, Oct. 2007.

[16] Y. E. Sagduyu and A. Ephremides, "Cross-layer optimization of MAC and network coding in wireless queueing tandem networks," IEEE Trans. on Inf. Theory, vol. 54, no. 2, pp. 554-571, Feb. 2008.

[17] A. Khreishah, C.-C. Wang, and N.-B. Shroff, “Cross-layer optimization for wireless multihop networks with pairwise intersession network coding," IEEE journal on Selected Areas in Commun., vol. 27, no. 5, pp. 606-621, Jun. 2009.

[18] J. Huang, V.-G. Subramanian, R. Agrawal, and R. Berry, "Downlink scheduling and resource allocation for OFDM systems," in Process of Conf. Info. Science Sys. (CISS), Mar. 2006.

[19] J. Huang, V.-G. Subramanian, R. Agrawal, and R. Berry, "Joint scheduling and resource allocation in uplink OFDM systems for broadband wireless access networks," in IEEE J. Sel. Top. Signal Process., vol. 27, no. 2, pp. 226-234, Feb. 2009.

[20] W. Yu, T. Kwon, and C. Shin, "Joint scheduling and dynamic power spectrum optimization for wireless multicell networks," in 
Proc. of 2010 44th Annual Conf. on Inf. Sciences and Systems (CISS' 2010), Princeton, New Jersey, USA,, pp. 1-6, Mar. 2010.

[21] L. Venturino, N. Prasad, and X. Wang, "Coordinated scheduling and power allocation in downlink multicell OFDMA networks," IEEE Trans. on Vehicular Tech., vol. 58, no. 6, pp. 2835-2848, Jul. 2009.

[22] Y. Shi, J. Zhang, and K.-B. Letaief, "Group sparse beamforming for green cloud-ran," IEEE Trans. on Wireless Commun., vol. 13, no. 5, pp. 2809-2823, May 2014.

[23] S. Kiani and D. Gesbert, "Optimal and distributed scheduling for multicell capacity maximization," IEEE Trans. on Wireless Commun., vol. 7, no. 1, pp. 288-297, Jan. 2008.

[24] W. Yu, T. Kwon, and C. Shin, "Multicell coordination via joint scheduling, beamforming, and power spectrum adaptation," IEEE Trans. Wireless Commun., vol. 12, no. 7, pp. 1-14, Jul. 2013.

[25] A. Stolyar and H. Viswanathan, "Self-organizing dynamic fractional frequency reuse for best-effort traffic through distributed inter-cell coordination," in Proc. 28th IEEE Conf. Comput. Commun. (INFOCOM'09), Rio de Janeiro, Brazil, pp. 1287-1295, Apr. 2009.

[26] R. Bendlin, Y.-F. Huang, M. Ivrlac, and J. Nossek, "Fast distributed multicell scheduling with delayed limited-capacity backhaul links," in Proc. IEEE Int. Conf. Commun. (ICC'09), Dresden, Germany, pp. 1-5, Jun. 2009.

[27] L. Jiang, S. Parekh, and J. Walrand, "Base station association game in multi-cell wireless networks (special paper)," in Proc. IEEE Wireless Commun. Netw. Conf. (WCNC'08), Las Vegas, Nevada, USA, pp. 1616-1621, Mar. 2008.

[28] R. Sun, M. Hong, and Z.-Q. Luo, "Optimal joint base station assignment and power allocation in a cellular network," in Proc. IEEE 13th Int. Workshop Signal Process. Adv. Wireless Commun. (SPAWC'12), Cesme, Turkey, pp. 234-238, Jun. 2012,

[29] J.-W. Lee, R. Mazumdar, and N. Shroff, "Joint resource allocation and base-station assignment for the downlink in CDMA networks," IEEE/ACM Trans. Netw., vol. 14, no. 1, pp. 1-14, Feb. 2006.

[30] A. Douik, H. Dahrouj, T.-Y. Al-Naffouri, and M.-S. Alouini, "Coordinated scheduling and power control in cloud-radio access networks," IEEE Trans. on Wireless Commun., vol. 15, no. 4, pp. 2523-2536, Apr. 2016.

[31] A. Douik, H. Dahrouj, T.-Y. Al-Naffouri, and M.-S. Alouini, "Lowcomplexity scheduling and power adaptation for coordinated cloud-radio access networks," IEEE Commun. Letters, vol. 21, no. 10, pp. 2298-2301, Oct. 2017.

[32] S. Katti, D. Katabi, W. Hu, H. Rahul, and M. Medard, "The importance of being opportunistic: practical network coding for wireless environments," in Proc. of Annual Allerton Conf. on Commun., Control and Computing (Allerton' 2005), Monticello, Illinois, USA, 2005.

[33] A. Afshin, M. Khabbazian, M. Ardakani, and G. Bansal "Blind instantly decodable network codes for wireless broadcast of realtime multimedia," IEEE Trans. on Wireless Commun., vol. 17, no. 4, pp. 2276-2288, Apr. 2018.

[34] L. Gou, G. Zhang, Z. Bian, D. Bian, and Z. Xie, "Minimizing completion time for relay-assisted multicast with instantly decodable network coding", IEEE Commun. Letters, vol. 20, pp. 434-437, Mar. 2016.

[35] L. Gou, G. Zhang, D. Bian, W. Zhang, and Z. Xie, “Data dissemination in wireless sensor networks with instantly decodable network coding", Journal of Commun. and Networks, vol. 18, pp. 846-856, Oct. 2016.

[36] S. Sorour and S. Valaee, "Completion delay minimization for instantly decodable network codes," IEEE/ACM Trans. on Netw., vol. 23, no. 5, pp. 1553-1567, Oct. 2015.

[37] A. Douik, S. Sorour, T.-Y. Al-Naffouri, and M.-S. Alouini, "Rate aware instantly decodable network codes," IEEE Trans. on Wireless Commun., vol. 16, no. 2, pp. 998-1011, Feb. 2017.

[38] X. Wang, C. Yuen, and Y. Xu, "Coding based data broadcasting for time critical applications with rate adaptation", IEEE Trans. on Vehicular Tech., vol. 63, no. 5, pp. 2429-2442, Jun. 2014.

[39] M.-S. Al-Abiad, A. Douik, and S. Sorour, "Rate aware network codes for cloud radio access networks," IEEE Trans. on Mobile Comp., vol. 18, no 8, pp 1898-1910, Aug. 2019.

[40] A. Le, A.-S. Tehrani, A.-G. Dimakis, and A. Markopoulou, "Instantly decodable network codes for real-time applications", in Proc. of 2013 Int. Symp. on Network Coding (NetCod), Calgary, AB, Canada, pp 1-6, Jun. 2013.

[41] M.-S. Karim, A. Douik, and S. Sorour, "Rate-aware network codes for video distortion reduction in point-to-multipoint net-
works,"IEEE Trans. on Vehicular Tech., vol. 66, no. 8, pp. 7446-7460, Aug. 2017.

[42] A. Douik and S. Sorour, "Data dissemination using instantly decodable binary codes in fog-radio access networks,"IEEE Trans. on Commun., vol. 66, no. 5, pp. 2052-2064, May 2018.

[43] H. Dahrouj, W. Yu, and T. Tang, "Power spectrum optimization for interference mitigation via iterative function evaluation," EURASIP Journal on Wireless Commun. and Networking, vol. 2012, no. 1, Dec. 2012.

[44] L.-P. Qian, Y. Zhang, and J. Huang, "Mapel: Achieving global optimality for a non-convex wireless power control problem," IEEE Trans. on Wireless Commun., vol. 8, no. 3, pp. 1553-1563, Mar. 2009.

[45] N. Bourgeois, B. Escoffier, V.-T. Paschos, and J-M.-M. van Rooij, "A bottom-up method and fast algorithms for max independent set," in Proc. of the 12th Scandinavian Conf. on Algorithm Theory (SWAT' 2010), Bergen, Norway.

[46] K. Yamaguchi and S. Masuda, "A new exact algorithm for the maximum weight clique problem," in Proc. Of the 23rd Int. Technical Conf. on Circuits/Systems, Computers and Commun. (ITCCSCC' 2008), Yamaguchi, Japan.

[47] P.-R.-J. Ostergard, "A fast algorithm for the maximum clique problem," Discrete Appl. Math, vol. 120, pp. 197-207.

[48] M.-R. Garey and D.-S. Johnson, Computers and intractability. Freeman New York, 2002, vol. 29.

[49] W. Yu, "Multiuser water-filling in the presence of crosstalk," in Proc. of Inf. Theory and Applications Workshop (ITA' 2007), San Diego, CA, USA, Jan. 2007, pp. 414-420.

[50] K. Shanmugam, N. Golrezaei, A. Dimakis, A. Molisch, and G. Caire, "Femtocaching: Wireless content delivery through distributed caching helpers," IEEE Trans. on Inf. Theory, vol. 59, no. 12, pp. 8402-8413, Dec. 2013. 